DESY-07-052

April 2007

\title{
Measurement of D mesons Production in Deep Inelastic Scattering at HERA
}

\author{
ZEUS Collaboration
}

\begin{abstract}
Charm production in deep inelastic scattering has been measured with the ZEUS detector at HERA using an integrated luminosity of $82 \mathrm{pb}^{-1}$. Charm has been tagged by reconstructing $D^{*+}, D^{0}, D^{+}$and $D_{s}^{+}$(+ c.c.) charm mesons. The charm hadrons were measured in the kinematic range $p_{T}\left(D^{*+}, D^{0}, D^{+}\right)>3 \mathrm{GeV}$, $p_{T}\left(D_{s}^{+}\right)>2 \mathrm{GeV}$ and $|\eta(D)|<1.6$ for $1.5<Q^{2}<1000 \mathrm{GeV}^{2}$ and $0.02<y<0.7$. The production cross sections were used to extract charm fragmentation ratios and the fraction of $c$ quarks hadronising into a particular charm meson in the kinematic range considered. The cross sections were compared to the predictions of next-to-leading-order QCD, and extrapolated to the full kinematic region in $p_{T}(D)$ and $\eta(D)$ in order to determine the open-charm contribution, $F_{2}^{c \bar{c}}\left(x, Q^{2}\right)$, to the proton structure function $F_{2}$.
\end{abstract}




\section{The ZEUS Collaboration}

S. Chekanov'ㄹ, M. Derrick, S. Magill, B. Musgrave, D. Nicholass'², J. Repond, R. Yoshida Argonne National Laboratory, Argonne, Illinois 60439-4815, USA ${ }^{n}$

M.C.K. Mattingly

Andrews University, Berrien Springs, Michigan 49104-0380, USA

M. Jechow, N. Pavel ${ }^{\dagger}$, A.G. Yagües Molina

Institut für Physik der Humboldt-Universität zu Berlin, Berlin, Germany

S. Antonelli, P. Antonioli, G. Bari, M. Basile, L. Bellagamba, M. Bindi, D. Boscherini,

A. Bruni, G. Bruni, L. Cifarelli, F. Cindolo, A. Contin, M. Corradi ${ }^{3}$, S. De Pasquale,

G. Iacobucci, A. Margotti, R. Nania, A. Polini, G. Sartorelli, A. Zichichi

University and INFN Bologna, Bologna, Italy ${ }^{e}$

D. Bartsch, I. Brock, S. Goers ${ }^{4}$, H. Hartmann, E. Hilger, H.-P. Jakob, M. Jüngst, O.M. Kind ${ }^{5}$,

A.E. Nuncio-Quiroz, E. Paul ${ }^{6}$, R. Renner ${ }^{4}$, U. Samson, V. Schönberg, R. Shehzadi, M. Wlasenko Physikalisches Institut der Universität Bonn, Bonn, Germany ${ }^{b}$

N.H. Brook, G.P. Heath, J.D. Morris, T. Namsoo

H.H. Wills Physics Laboratory, University of Bristol, Bristol, United Kingdom ${ }^{m}$

M. Capua, S. Fazio, A. Mastroberardino, M. Schioppa, G. Susinno, E. Tassi

Calabria University, Physics Department and INFN, Cosenza, Italy ${ }^{e}$

J.Y. $\operatorname{Kim}^{7}$, K.J. Ma ${ }^{8}$

Chonnam National University, Kwangju, South Korea ${ }^{g}$

Z.A. Ibrahim, B. Kamaluddin, W.A.T. Wan Abdullah

Jabatan Fizik, Universiti Malaya, 50603 Kuala Lumpur, Malaysia ${ }^{r}$

Y. Ning, Z. Ren, F. Sciulli

Nevis Laboratories, Columbia University, Irvington on Hudson, New York 10027o

J. Chwastowski, A. Eskreys, J. Figiel, A. Galas, M. Gil, K. Olkiewicz, P. Stopa, L. Zawiejski

The Henryk Niewodniczanski Institute of Nuclear Physics, Polish Academy of Sciences, Cracow, Poland ${ }^{i}$

L. Adamczyk, T. Bołd, I. Grabowska-Bołd, D. Kisielewska, J. Łukasik, M. Przybycień,

L. Suszycki

Faculty of Physics and Applied Computer Science, AGH-University of Science and Technology, Cracow, Poland ${ }^{p}$

A. Kotański ${ }^{9}$, W. Słomiński ${ }^{10}$

Department of Physics, Jagellonian University, Cracow, Poland 
V. Adler ${ }^{4}$, U. Behrens, I. Bloch, C. Blohm, A. Bonato, K. Borras, R. Ciesielski, N. Coppola, A. Dossanov, V. Drugakov, J. Fourletova, A. Geiser, D. Gladkov, P. Göttlicher ${ }^{11}$, J. Grebenyuk, I. Gregor, T. Haas, W. Hain, C. Horn ${ }^{12}$, A. Hüttmann, B. Kahle, I.I. Katkov, U. Klein ${ }^{13}$, U. Kötz, H. Kowalski, E. Lobodzinska, B. Löhr, R. Mankel, I.-A. MelzerPellmann, S. Miglioranzi, A. Montanari, D. Notz, L. Rinaldi, P. Roloff, I. Rubinsky, R. Santamarta, U. Schneekloth, A. Spiridonov ${ }^{14}$, H. Stadie, D. Szuba ${ }^{15}$, J. Szuba ${ }^{16}$, T. Theedt, G. Wolf, K. Wrona, C. Youngman, W. Zeuner Deutsches Elektronen-Synchrotron DESY, Hamburg, Germany

W. Lohmann, S. Schlenstedt

Deutsches Elektronen-Synchrotron DESY, Zeuthen, Germany

G. Barbagli, E. Gallo, P. G. Pelfer

University and INFN, Florence, Italy ${ }^{e}$

A. Bamberger, D. Dobur, F. Karstens, N.N. Vlasov ${ }^{17}$

Fakultät für Physik der Universität Freiburg i.Br., Freiburg i.Br., Germany ${ }^{b}$

P.J. Bussey, A.T. Doyle, W. Dunne, J. Ferrando, M. Forrest, D.H. Saxon, I.O. Skillicorn

Department of Physics and Astronomy, University of Glasgow, Glasgow, United Kingdom $^{m}$

I. Gialas ${ }^{18}$, K. Papageorgiu

Department of Engineering in Management and Finance, Univ. of Aegean, Greece

T. Gosau, U. Holm, R. Klanner, E. Lohrmann, H. Salehi, P. Schleper, T. Schörner-Sadenius, J. Sztuk, K. Wichmann, K. Wick

Hamburg University, Institute of Exp. Physics, Hamburg, Germany ${ }^{b}$

C. Foudas, C. Fry, K.R. Long, A.D. Tapper

Imperial College London, High Energy Nuclear Physics Group, London, United Kingdom $^{m}$

M. Kataoka ${ }^{19}$, T. Matsumoto, K. Nagano, K. Tokushuku ${ }^{20}$, S. Yamada, Y. Yamazaki Institute of Particle and Nuclear Studies, KEK, Tsukuba, Japan ${ }^{f}$

A.N. Barakbaev, E.G. Boos, N.S. Pokrovskiy, B.O. Zhautykov

Institute of Physics and Technology of Ministry of Education and Science of Kazakhstan, Almaty, Kazakhstan

D. Son

Kyungpook National University, Center for High Energy Physics, Daegu, South Korea ${ }^{g}$

J. de Favereau, K. Piotrzkowski

Institut de Physique Nucléaire, Université Catholique de Louvain, Louvain-la-Neuve, Belgium $^{q}$ 
F. Barreiro, C. Glasman ${ }^{21}$, M. Jimenez, L. Labarga, J. del Peso, E. Ron, M. Soares, J. Terrón, M. Zambrana

Departamento de Física Teórica, Universidad Autónoma de Madrid, Madrid, Spain ${ }^{l}$

F. Corriveau, C. Liu, R. Walsh, C. Zhou

Department of Physics, McGill University, Montréal, Québec, Canada H3A 2T8 ${ }^{a}$

T. Tsurugai

Meiji Gakuin University, Faculty of General Education, Yokohama, Japan ${ }^{f}$

A. Antonov, B.A. Dolgoshein, V. Sosnovtsev, A. Stifutkin, S. Suchkov

Moscow Engineering Physics Institute, Moscow, Russia ${ }^{j}$

R.K. Dementiev, P.F. Ermolov, L.K. Gladilin, L.A. Khein, I.A. Korzhavina, V.A. Kuzmin, B.B. Levchenko ${ }^{22}$, O.Yu. Lukina, A.S. Proskuryakov, L.M. Shcheglova, D.S. Zotkin, S.A. Zotkin

Moscow State University, Institute of Nuclear Physics, Moscow, Russia ${ }^{k}$

I. Abt, C. Büttner, A. Caldwell, D. Kollar, W.B. Schmidke, J. Sutiak

Max-Planck-Institut für Physik, München, Germany

G. Grigorescu, A. Keramidas, E. Koffeman, P. Kooijman, A. Pellegrino, H. Tiecke, M. Vázquez ${ }^{19}$, L. Wiggers

NIKHEF and University of Amsterdam, Amsterdam, Netherlands ${ }^{h}$

N. Brümmer, B. Bylsma, L.S. Durkin, A. Lee, T.Y. Ling

Physics Department, Ohio State University, Columbus, Ohio 43210 ${ }^{n}$

P.D. Allfrey, M.A. Bell, A.M. Cooper-Sarkar, A. Cottrell, R.C.E. Devenish, B. Foster, K. Korcsak-Gorzo, S. Patel, V. Roberfroid ${ }^{23}$, A. Robertson, P.B. Straub, C. UribeEstrada, R. Walczak

Department of Physics, University of Oxford, Oxford United Kingdom ${ }^{m}$

P. Bellan, A. Bertolin, R. Brugnera, R. Carlin, F. Dal Corso, S. Dusini, A. Garfagnini,

S. Limentani, A. Longhin, L. Stanco, M. Turcato

Dipartimento di Fisica dell' Università and INFN, Padova, Italy ${ }^{e}$

B.Y. Oh, A. Raval, J. Ukleja ${ }^{24}$, J.J. Whitmore ${ }^{25}$

Department of Physics, Pennsylvania State University, University Park, Pennsylvania $16802^{\circ}$

Y. Iga

Polytechnic University, Sagamihara, Japan ${ }^{f}$

G. D'Agostini, G. Marini, A. Nigro

Dipartimento di Fisica, Università 'La Sapienza' and INFN, Rome, Italy ${ }^{e}$ 
J.E. Cole, J.C. Hart

Rutherford Appleton Laboratory, Chilton, Didcot, Oxon, United Kingdom ${ }^{m}$

H. Abramowicz ${ }^{26}$, A. Gabareen, R. Ingbir, S. Kananov, A. Levy

Raymond and Beverly Sackler Faculty of Exact Sciences, School of Physics, Tel-Aviv

University, Tel-Aviv, Israel ${ }^{d}$

M. Kuze, J. Maeda

Department of Physics, Tokyo Institute of Technology, Tokyo, Japan ${ }^{f}$

R. Hori, S. Kagawa ${ }^{27}$, N. Okazaki, S. Shimizu, T. Tawara

Department of Physics, University of Tokyo, Tokyo, Japan ${ }^{f}$

R. Hamatsu, H. Kaji ${ }^{28}$, S. Kitamura ${ }^{29}$, O. Ota, Y.D. Ri

Tokyo Metropolitan University, Department of Physics, Tokyo, Japan ${ }^{f}$

M.I. Ferrero, V. Monaco, R. Sacchi, A. Solano

Università di Torino and INFN, Torino, Italy ${ }^{e}$

M. Arneodo, M. Ruspa

Università del Piemonte Orientale, Novara, and INFN, Torino, Italy ${ }^{e}$

S. Fourletov, J.F. Martin

Department of Physics, University of Toronto, Toronto, Ontario, Canada M5S 1A7 a

S.K. Boutle ${ }^{18}$, J.M. Butterworth, C. Gwenlan ${ }^{30}$, T.W. Jones, J.H. Loizides, M.R. Sutton ${ }^{30}$, M. Wing

Physics and Astronomy Department, University College London, London, United Kingdom $^{m}$

B. Brzozowska, J. Ciborowski ${ }^{31}$, G. Grzelak, P. Kulinski, P. Łużniak ${ }^{32}$, J. Malka ${ }^{32}$, R.J. Nowak, J.M. Pawlak, T. Tymieniecka, A. Ukleja, A.F. Żarnecki

Warsaw University, Institute of Experimental Physics, Warsaw, Poland

M. Adamus, P. Plucinski ${ }^{33}$

Institute for Nuclear Studies, Warsaw, Poland

Y. Eisenberg, I. Giller, D. Hochman, U. Karshon, M. Rosin

Department of Particle Physics, Weizmann Institute, Rehovot, Israel ${ }^{c}$

E. Brownson, T. Danielson, A. Everett, D. Kçira, D.D. Reeder ${ }^{6}$, P. Ryan, A.A. Savin, W.H. Smith, H. Wolfe

Department of Physics, University of Wisconsin, Madison, Wisconsin 53706, USA ${ }^{n}$

S. Bhadra, C.D. Catterall, Y. Cui, G. Hartner, S. Menary, U. Noor, J. Standage, J. Whyte

Department of Physics, York University, Ontario, Canada M3J 1Pз ${ }^{a}$ 
${ }^{1}$ supported by DESY, Germany

2 also affiliated with University College London, UK

3 also at University of Hamburg, Germany, Alexander von Humboldt Fellow

${ }^{4}$ self-employed

${ }^{5}$ now at Humboldt University, Berlin, Germany

${ }^{6}$ retired

7 supported by Chonnam National University in 2005

${ }^{8}$ supported by a scholarship of the World Laboratory Björn Wiik Research Project

9 supported by the research grant no. 1 P03B 04529 (2005-2008)

10 This work was supported in part by the Marie Curie Actions Transfer of Knowledge project COCOS (contract MTKD-CT-2004-517186)

11 now at DESY group FEB, Hamburg, Germany

12 now at Stanford Linear Accelerator Center, Stanford, USA

13 now at University of Liverpool, UK

14 also at Institut of Theoretical and Experimental Physics, Moscow, Russia

15 also at INP, Cracow, Poland

16 on leave of absence from FPACS, AGH-UST, Cracow, Poland

17 partly supported by Moscow State University, Russia

18 also affiliated with DESY

19 now at CERN, Geneva, Switzerland

20 also at University of Tokyo, Japan

${ }^{21}$ Ramón y Cajal Fellow

22 partly supported by Russian Foundation for Basic Research grant no. 05-02-39028NSFC-a

${ }^{23}$ EU Marie Curie Fellow

${ }^{24}$ partially supported by Warsaw University, Poland

25 This material was based on work supported by the National Science Foundation, while working at the Foundation.

26 also at Max Planck Institute, Munich, Germany, Alexander von Humboldt Research Award

27 now at KEK, Tsukuba, Japan

28 now at Nagoya University, Japan

29 Department of Radiological Science

30 PPARC Advanced fellow

31 also at Łódź University, Poland

32 Łódź University, Poland

${ }^{33}$ supported by the Polish Ministry for Education and Science grant no. 1 P03B 14129

$\dagger$ deceased 
a supported by the Natural Sciences and Engineering Research Council of Canada (NSERC)

$b$ supported by the German Federal Ministry for Education and Research (BMBF), under contract numbers HZ1GUA 2, HZ1GUB 0, HZ1PDA 5, HZ1VFA 5

c supported in part by the MINERVA Gesellschaft für Forschung GmbH, the Israel Science Foundation (grant no. 293/02-11.2) and the U.S.-Israel Binational Science Foundation

$d \quad$ supported by the German-Israeli Foundation and the Israel Science Foundation

$e$ supported by the Italian National Institute for Nuclear Physics (INFN)

$f$ supported by the Japanese Ministry of Education, Culture, Sports, Science and Technology (MEXT) and its grants for Scientific Research

$g$ supported by the Korean Ministry of Education and Korea Science and Engineering Foundation

$h$ supported by the Netherlands Foundation for Research on Matter (FOM)

$i$ supported by the Polish State Committee for Scientific Research, grant no. 620/E-77/SPB/DESY/P-03/DZ 117/2003-2005 and grant no. 1P03B07427/2004-2006

j partially supported by the German Federal Ministry for Education and Research $(\mathrm{BMBF})$

$k$ supported by RF Presidential grant N 8122.2006.2 for the leading scientific schools and by the Russian Ministry of Education and Science through its grant Research on High Energy Physics

$l$ supported by the Spanish Ministry of Education and Science through funds provided by CICYT

$m$ supported by the Particle Physics and Astronomy Research Council, UK

$n$ supported by the US Department of Energy

$o$ supported by the US National Science Foundation. Any opinion, findings and conclusions or recommendations expressed in this material are those of the authors and do not necessarily reflect the views of the National Science Foundation.

$p$ supported by the Polish Ministry of Science and Higher Education as a scientific project (2006-2008)

$q$ supported by FNRS and its associated funds (IISN and FRIA) and by an Inter-University Attraction Poles Programme subsidised by the Belgian Federal Science Policy Office

$r$ supported by the Malaysian Ministry of Science, Technology and Innovation/Akademi Sains Malaysia grant SAGA 66-02-03-0048 


\section{Introduction}

Heavy-quark production in ep interactions in the deep inelastic scattering (DIS) regime is dominated by the interaction between the exchanged virtual photon and a gluon within the proton, the so-called Boson Gluon Fusion (BGF) mechanism. Heavy-quark production provides a twofold test of perturbative quantum chromodinamics (pQCD): a study of the BGF process and the higher order corrections to it, and an independent check of the validity of the gluon density in the proton extracted from the inclusive DIS data. Of the two heavy quarks whose production is accessible by HERA, $c$ and $b$, the latter is strongly suppressed due to its smaller electric charge and larger mass. This paper reports a study of $c$-quark production.

A charm quark in the final state is identified by the presence of a corresponding charmed hadron. This paper studies the production of the pseudo-scalar mesons $D^{0}, D^{+}, D_{s}^{+}$and the vector meson $D^{*+}$ from the decays $D^{0} \rightarrow K^{-} \pi^{+}, D^{+} \rightarrow K^{-} \pi^{+} \pi^{+}, D_{s}^{+} \rightarrow \phi \pi^{+} \rightarrow$ $K^{+} K^{-} \pi^{+}$and $D^{*+} \rightarrow D^{0} \pi^{+} \rightarrow K^{-} \pi^{+} \pi^{+}$(the charge conjugated modes are implied throughout this paper). Since a $D$ hadron is measured and not the $c$ quark itself, any comparison with pQCD requires a modelling of the $c \rightarrow D$ fragmentation. A consequence of the QCD factorisation theorem [1] is that the "hard" (pQCD governed) c-production mechanism is independent of the "soft" fragmentation process. Measurements of $D$ hadron cross sections provide therefore information about both $c$-quark production and its fragmentation.

This paper presents a complete study of $D$-meson production in DIS at HERA: measurements of $c$-quark fragmentation ratios and fractions with unprecedent precision, $D$-meson differential cross sections and the charm contribution, $F_{2}^{c \bar{c}}$, to the proton structure function $F_{2}$. It addresses the universality of fragmentation and tests the predictions of pQCD for charm production. The data sample used was taken by the ZEUS detector during the years 1998 - 2000. The fragmentation measurements follow closely those reported recently by ZEUS in the photoproduction regime [2]. Using a variety of $D$ mesons, the pQCD analysis complements the study done with $D^{*+}$ in the same data sample [3]. Measurements of $D^{*}$ cross sections are only used in this paper for the extraction of the fragmentation parameters.

Similar measurements of the properties of $c$-quark fragmentation in DIS have also been performed by the H1 collaboration [4]. Other previous measurements of charm production in DIS with pQCD analyses used the $D^{*+}$ meson [3,5-9] or inclusive lifetime tags [10]. There are also several measurements of charm photoproduction [9,11-15]. 


\section{Experimental set-up}

The analysis was performed with data taken from 1998 to 2000, when HERA collided electrons or positrons with energy $E_{e}=27.5 \mathrm{GeV}$ on protons of energy $E_{p}=920 \mathrm{GeV}$. The results are based on $e^{-} p$ and $e^{+} p$ samples corresponding to integrated luminosities of $16.7 \pm 0.3 \mathrm{pb}^{-1}$ and $65.1 \pm 1.5 \mathrm{pb}^{-1}$, respectively. ${ }^{1}$

A detailed description of the ZEUS detector can be found elsewhere [16]. A brief outline of the components that are most relevant for this analysis is given below.

Charged particles are tracked in the central tracking detector (CTD) [17], which operates in a magnetic field of $1.43 \mathrm{~T}$ provided by a thin superconducting coil. The CTD consists of 72 cylindrical drift chamber layers, organised in 9 superlayers covering the polar-angle ${ }^{2}$ region $15^{\circ}<\theta<164^{\circ}$. The transverse-momentum resolution for full-length tracks is $\sigma\left(p_{T}\right) / p_{T}=0.0058 p_{T} \oplus 0.0065 \oplus 0.0014 / p_{T}$, with $p_{T}$ in $\mathrm{GeV}$.

The high-resolution uranium-scintillator calorimeter (CAL) [18] consists of three parts: the forward (FCAL), the barrel (BCAL) and the rear (RCAL) calorimeters. Each part is subdivided transversely into towers and longitudinally into one electromagnetic section and either one (in RCAL) or two (in BCAL and FCAL) hadronic sections. The smallest subdivision of the calorimeter is called a cell. The CAL energy resolutions, as measured under test-beam conditions, are $\sigma(E) / E=0.18 / \sqrt{E}$ for electrons and $\sigma(E) / E=0.35 / \sqrt{E}$ for hadrons, with $E$ in $\mathrm{GeV}$.

The position of the scattered electron was determined by combining information from the CAL, the small-angle rear tracking detector (SRTD) [19] and the hadron-electron separator (HES) [20].

The luminosity was measured from the rate of the bremsstrahlung process $e p \rightarrow e \gamma p$, where the photon was measured in a lead-scintillator calorimeter [21] placed in the HERA tunnel at $Z=-107 \mathrm{~m}$.

\section{Theoretical predictions}

The next-to-leading order (NLO) QCD predictions for $c \bar{c}$ cross sections were obtained using the HVQDIS program [22] based on the so-called fixed-flavour-number scheme (FFNS). In this scheme, only light partons $(u, d, s, g)$ are included in the proton parton density

\footnotetext{
${ }^{1}$ Hereafter, both electrons and positrons are referred to as electrons, unless explicitly stated otherwise.

${ }^{2}$ The ZEUS coordinate system is a right-handed Cartesian system, with the $Z$ axis pointing in the proton beam direction, referred to as the "forward direction", and the $X$ axis pointing left towards the centre of HERA. The coordinate origin is at the nominal interaction point.
} 
functions (PDFs) which obey the DGLAP equations [23], and the $c \bar{c}$ pair is produced via the BGF mechanism [24] with NLO corrections [25]. This calculation is expected to be valid [26] in the kinematic range of this measurement, $1.5<Q^{2}<1000 \mathrm{GeV}^{2}$, where $Q^{2}$ is the negative of the four-momentum transfer squared, hereafter referred to as photon virtuality.

The following inputs have been used to obtain the predictions for $D$-meson production at NLO using the program HVQDIS. The FFNS variant of the ZEUS-S NLO QCD fit [27] to structure-function data was used as the parameterisation of the proton PDFs. In this fit the three-flavour QCD scale $\Lambda_{\mathrm{QCD}}$ was set to $\Lambda_{\mathrm{QCD}}^{(3)}=0.363 \mathrm{GeV}$ and the mass of the charm quark was set to $1.35 \mathrm{GeV}$; the same mass and $\Lambda_{\mathrm{QCD}}^{(3)}$ were therefore used in the HVQDIS calculation. The renormalisation and factorisation scales were set to $\mu=\sqrt{Q^{2}+4 m_{c}^{2}}$ for charm production both in the fit and in the HVQDIS calculation. The charm fragmentation to the particular $D$ meson was carried out using the Peterson function [28]. The values used for the hadronisation fractions to $D$ mesons, $f(c \rightarrow D)$, were those measured in this paper, and the Peterson parameter, $\epsilon$, was set to 0.035 [29]. The effect of $J / \psi$ production was found to be negligible [30,31].

\section{Kinematic reconstruction and event selection}

The kinematic variables $Q^{2}$, the Bjorken scaling variable, $x$ (in the quark-parton model $x$ can be interpreted as the fraction of proton momentum carried by the struck quark), and the fraction of the electron energy transferred to the proton in the rest frame of the proton, $y$, can be reconstructed using a variety of methods, whose accuracy depends on the variable of interest and its range:

- for the electron method (specified with the subscript $e$ ), the measured energy and angle of the scattered electron are used;

- the double angle (DA) method [32] relies on the angles of the scattered electron and of the hadronic system;

- the Jacquet-Blondel (JB) method [33] is based entirely on measurements of the hadronic system;

- the $\Sigma$-method [34] uses both the scattered-electron energy and angle, and measurements of the hadronic system.

The reconstruction of $Q^{2}$ and $x$ was performed using the $\Sigma$-method, since it has better resolution at low $Q^{2}$ than the DA method. At high $Q^{2}$, the $\Sigma$-method and the DA method are similar, and both have better resolution than the electron method. The DA method was used as a systematic check. 
A three-level trigger system was used to select events online $[16,35]$. At the third level, events having at least a reconstructed $D^{*+}, D^{0}, D^{+}$(only $e^{+} p$ sample), $D_{s}^{+}$or $\Lambda_{c}^{+}$candidate, as well as a scattered-electron candidate, were kept for further analysis. The efficiency of the online reconstruction for any of the above hadrons, determined relative to an inclusive DIS trigger, was generally above $95 \%$.

The events were selected offline $[5,36]$ using the following cuts:

- the scattered electron was identified using a neural-network procedure $[37,38]$. Its energy, $E_{e^{\prime}}$, was required to be larger than $10 \mathrm{GeV}$;

- $y_{e} \leq 0.95$ (where $y_{e}$ is $y$ reconstructed with the electron method) and $y_{\mathrm{JB}} \geq 0.02$ (where $y_{\mathrm{JB}}$ is $y$ reconstructed with the JB method). The former condition removes events where fake electrons are found in the FCAL and the latter rejects events where the hadronic system cannot be measured precisely, in order to reconstruct the kinematic variables;

- $40 \leq \delta \leq 65 \mathrm{GeV}$, where $\delta=\sum E_{i}\left(1-\cos \theta_{i}\right)$ and $E_{i}$ and $\theta_{i}$ are the energy and the polar angle of the $i^{\text {th }}$ energy-flow object (EFO) [39] reconstructed from charged tracks, as measured in the CTD, and energy clusters measured in the CAL. The sum $i$ runs over all EFOs;

- a primary vertex position determined from the tracks fitted to the vertex in the range $\left|Z_{\text {vertex }}\right|<50 \mathrm{~cm}$

- the impact point $(X, Y)$ of the scattered electron on the RCAL was required to lie outside the region $26 \times 14 \mathrm{~cm}^{2}$ centred on $X=Y=0$.

The angle of the scattered electron was determined using either its impact position on the CAL inner face or a reconstructed track in the CTD. When available, SRTD and HES were also used. The energy of the scattered electron was corrected for non-uniformity effects caused by cell and module boundaries.

The selected kinematic region was $1.5<Q^{2}<1000 \mathrm{GeV}^{2}$ and $0.02<y<0.7$.

\section{Reconstruction of charm hadrons}

The production of $D^{*+}, D^{0}, D^{+}$, and $D_{s}^{+}$charm mesons was measured in the range of transverse momentum $p_{T}(D)>3 \mathrm{GeV}$ and pseudorapidity $|\eta(D)|<1.6$. For the $D_{s}^{+}$, the $p_{T}\left(D_{s}^{+}\right)$requirement was relaxed to $p_{T}\left(D_{s}^{+}\right)>2 \mathrm{GeV}$, as the constraint provided by the $K^{+} K^{-}$pair coming from a $\phi$ meson kept the combinatorial background at acceptable levels. The reconstruction of the $\Lambda_{c}^{+}$baryon was attempted using the decay $\Lambda_{c}^{+} \rightarrow K^{-} p \pi^{+}$. 
The signal achieved had a statistical significance of around three standard deviations, and therefore it was not used.

The charm mesons were reconstructed using tracks measured in the CTD and assigned to the reconstructed event vertex. To ensure good momentum resolution, each track was required to reach at least the third superlayer of the CTD. Further background reduction was achieved by imposing cuts on the transverse momenta and decay angles of the charm-hadron decay products. The cut values were optimised using Monte Carlo (MC) simulation to enhance signal over background ratios while keeping acceptances high.

The cross sections of the $D^{0}$ and $D^{*+}$ mesons and related quantities involved in the measurements of fragmentation properties can be obtained from the combination of three independent samples [2]: those of $D^{0}$ candidates with and without a " $\Delta M$ " tag and that of "additional" $D^{*+}$ candidates. The samples are described below. The rationale for this division [2] will become apparent in Sections 8 and 9.

\subsection{Reconstruction of $D^{0}$ mesons}

The $D^{0}$ mesons were reconstructed from the decay channel $D^{0} \rightarrow K^{-} \pi^{+}$. In each event, $D^{0}$ candidates were formed from pairs of tracks with opposite charges and $p_{T}>0.8 \mathrm{GeV}$. The nominal kaon and pion masses were assumed in turn for each track. To reduce the combinatorial background, a further cut was applied in the angle, $\theta^{*}(K)$, between the kaon in the $D$-candidate rest frame and the $D$-candidate line of flight in the laboratory frame, $\left|\cos \theta^{*}(K)\right|<0.85$. The $D^{0}$ candidates were separated into two groups. The $\Delta M$ tag group consists of $D^{0}$ candidates that, when combined with a third track that could be a "soft" pion $\left(\pi_{s}\right)$ in a $D^{*+} \rightarrow D^{0} \pi_{s}^{+}$decay, have $\Delta M=M\left(K \pi \pi_{s}\right)-M(K \pi)$ in the range $0.143<\Delta M<0.148 \mathrm{GeV}$. The soft pion was required to have $p_{T}>0.2 \mathrm{GeV}$ and charge opposite to that of the kaon. This cut was raised to $p_{T}>0.25 \mathrm{GeV}$ for a data subsample, corresponding to an integrated luminosity of $17 \mathrm{pb}^{-1}$, for which the track reconstruction efficiency at low momentum was smaller due to the operating conditions of the CTD [40]. For the untagged $D^{0}$ candidates, the incorrect assignment of the pion and kaon masses to the two tracks produces a wider reflected signal. This reflection was estimated from the $D^{0}$ candidates with a $\Delta M$ tag and normalised to the ratio of numbers of $D^{0}$ without and with $\Delta M$ tag; it was then subtracted from the untagged $D^{0}$ candidates.

Figure 1 shows the $M(K \pi)$ distributions for untagged $D^{0}$ candidates after the reflection subtraction and for tagged $D^{0}$ candidates. The distributions were fitted simultaneously assuming that both have the same shape and are described by the "modified" Gaussian function [2]: 


$$
\operatorname{Gauss}^{\bmod }\left(M_{0}, \sigma\right) \propto e^{\left[-0.5 \cdot x^{1+1 /(1+0.5 \cdot x)}\right]},
$$

where $x=\left|\left[M\left(K^{-} \pi^{+}\right)-M_{0}\right] / \sigma\right|$. The background shape in the fit was described [2] by the form $\left[A+B \cdot M\left(K^{-} \pi^{+}\right)\right]$for $M\left(K^{-} \pi^{+}\right)>1.86 \mathrm{GeV}$ and $\left[A+B \cdot M\left(K^{-} \pi^{+}\right)\right] \cdot \exp \{C$. $\left.\left[M\left(K^{-} \pi^{+}\right)-1.86\right]\right\}$ for $M\left(K^{-} \pi^{+}\right)<1.86 \mathrm{GeV}$. The free parameters $A, B$ and $C$ were assumed to be independent for the two $M\left(K^{-} \pi^{+}\right)$distributions. Clear signals are seen at the nominal value of $M\left(D^{0}\right)$ [41]. The number of untagged (tagged) $D^{0}$ mesons yielded by the fit was $N^{\text {untag }}\left(D^{0}\right)=7996 \pm 488\left(N^{\operatorname{tag}}\left(D^{0}\right)=1970 \pm 78\right)$.

\subsection{Reconstruction of "additional" $D^{*+}$ mesons}

The $D^{*+} \rightarrow D^{0} \pi_{s}^{+}$events with $p_{T}\left(D^{*+}\right)>3 \mathrm{GeV}$ and $\left|\eta\left(D^{*+}\right)\right|<1.6$ can be considered as a sum of two subsamples: events with the $D^{0}$ having $p_{T}\left(D^{0}\right)>3 \mathrm{GeV}$ and $\left|\eta\left(D^{0}\right)\right|<1.6$, and events with the $D^{0}$ outside of that kinematic range. The former sample is a subset of $D^{0}$ mesons reconstructed with $\Delta M$ tag, as discussed above. The latter sample of additional $D^{*+}$ mesons was obtained using the same $D^{0} \rightarrow K^{-} \pi^{+}$decay channel. In each event, pairs of tracks with $p_{T}>0.4 \mathrm{GeV}$ were combined to form a $D^{0}$ candidate. Only combinations with invariant mass $1.80<M(K \pi)<1.92 \mathrm{GeV}$ were considered. The $D^{0}$ candidates were required to have $p_{T}\left(D^{0}\right)<3.0 \mathrm{GeV}$ or $\left|\eta\left(D^{0}\right)\right|>1.6$. A third track with $p_{T}>0.2 \mathrm{GeV}$, with charge opposite to that of the kaon track and assumed to have the pion mass, was combined with the $D^{0}$ candidate to form an additional $D^{* \pm}$ candidate. Here again the cut value was $p_{T}>0.25 \mathrm{GeV}$ for the data subsample for which the track reconstruction efficiency at low momentum was smaller.

Figure 2 shows the distribution of the mass difference $\Delta M=M\left(K \pi \pi_{s}\right)-M(K \pi)$ for additional $D^{*}$ candidates. A clear signal is seen around the nominal value of $M\left(D^{* \pm}\right)-$ $M\left(D^{0}\right)[41]$. The combinatorial background under the signal was estimated from the massdifference distribution of the wrong-charge combinations, in which both tracks associated to the $D^{0}$ candidate have the same charge and the third track has opposite charge. The number of reconstructed additional $D^{*+}$ mesons was calculated by subtracting the wrongcharge $\Delta M$ distribution, after normalising it to the number of right-charge candidates in the region $0.150<\Delta M<0.170 \mathrm{GeV}$. The subtraction was done in the signal region $0.143<\Delta M<0.148 \mathrm{GeV}$ and yielded $N^{\text {add }}\left(D^{*+}\right)=317 \pm 26$.

\subsection{Reconstruction of $D^{+}$mesons}

The $D^{+}$mesons were reconstructed from the decay channel $D^{+} \rightarrow K^{-} \pi^{+} \pi^{+}$. The analysis for this meson was done using the $e^{+} p$ data sample only, where the $D^{+}$third level 
trigger logic was implemented. In each event, two tracks with the same charge and a third track with opposite charge were combined to form a $D^{+}$candidate. The tracks with the same charge were assigned the pion mass and required to have $p_{T}(\pi)>0.5 \mathrm{GeV}$. For the remaining track, the kaon mass was assumed and $p_{T}(K)>0.7 \mathrm{GeV}$ was required. Combinatorial background was further suppressed by requiring $\cos \theta^{*}(K)>-0.75$. Background from $D^{*+}$ and $D_{s}^{+} \rightarrow \phi \pi^{+}$with $\phi \rightarrow K^{+} K^{-}$was suppressed by applying suitable cuts [2].

Figure 3 shows the $M\left(K^{-} \pi^{+} \pi^{+}\right)$distribution for the $D^{+}$candidates. A clear signal is seen at the nominal value of $D^{+}$mass [41]. The mass distribution was fitted to a sum of the modified Gaussian function (Eq. (1)) describing the signal and a linear function describing the non-resonant background. The number of reconstructed $D^{+}$mesons yielded by the fit was $N\left(D^{+}\right)=4785 \pm 501$.

\subsection{Reconstruction of $D_{s}^{+}$mesons}

The $D_{s}^{+}$mesons were reconstructed from the decay channel $D_{s}^{+} \rightarrow \phi \pi^{+}$with $\phi \rightarrow K^{+} K^{-}$. In each event, two tracks with opposite charges were each assigned the kaon mass and combined to form the $\phi$ candidates if the invariant mass $M(K K)$ was within $\pm 8 \mathrm{MeV}$ of the nominal $\phi$ mass [2]. A third track, assumed to be a pion, was combined with the $\phi$ candidate, yielding the $D_{s}^{+}$candidate. Only tracks with $p_{T}(\pi)>0.5 \mathrm{GeV}$ and $p_{T}(K)>0.7 \mathrm{GeV}$ were considered. To reduce the combinatorial background further, a cut was applied in the angle, $\theta^{*}(\pi)$, between the pion in the $K K \pi$ rest frame and the $K K \pi$ line of flight in the laboratory frame, $\cos \theta^{*}(\pi)<0.85$, and in the angle, $\theta^{\prime}(K)$, between one of the kaons and the pion in the $K K$ rest frame, $\left|\cos ^{3} \theta^{\prime}(K)\right|>0.1$.

Figure 4 shows the $M\left(K^{+} K^{-} \pi^{+}\right)$distributions for the $D_{s}^{+}$candidates after all cuts. A clear signal is seen at the nominal $D_{s}^{+}$mass [41]. There is also a smaller signal around the nominal $D^{+}$mass as expected from the decay $D^{+} \rightarrow \phi \pi^{+}$. The mass distribution was fitted to a sum of two modified Gaussian functions (Eq. (1)) describing the signals and an exponential function describing the non-resonant background. The number of reconstructed $D_{s}^{+}$mesons yielded by the fit was $N\left(D_{s}^{+}\right)=647 \pm 80$, for $p_{T}\left(D_{s}^{+}\right)>3 \mathrm{GeV}$ and $N\left(D_{s}^{+}\right)=773 \pm 96$, for $p_{T}\left(D_{s}^{+}\right)>2 \mathrm{GeV}$.

\section{Acceptance corrections}

The acceptances were calculated using the RAPGAP 2.08 [42] MC model and checked with Herwig 6.3 [43]. The RAPGAP MC model was interfaced with Heracles 4.6.1 [44] in order to incorporate first-order electroweak corrections. The generated events were then 
passed through a full simulation of the detector, using GEANT 3.13 [45], processed and selected with the same programs as used for the data.

The MC models were used to produce charm by the BGF and the resolved photon processes. In the latter the virtual photon behaves as a hadron-like source of partons, one of which interacts with a parton of the initial proton. The CTEQ5L [46] and GRV-LO [47] PDFs were used for the proton and the photon, respectively. The charm-quark mass was set to $1.5 \mathrm{GeV}$. Both the RAPGAP and Herwig MCs use LO matrix elements with leading-logarithmic parton showers. Charm fragmentation is implemented using either the Lund string fragmentation (in RAPGAP) or a cluster fragmentation [48] model (in HeRWIG). It was checked that both MC samples, RAPGAP and HerwiG, give a reasonable description of the data for DIS and $D$-meson variables when compared at detector-level.

For a given observable $Y$, the production differential cross sections were determined using

$$
\frac{d \sigma}{d Y}=\frac{N(D)}{\mathcal{A} \cdot \mathcal{L} \cdot \mathcal{B} \cdot \Delta Y}
$$

where $N(D)$ is the number of reconstructed $D$ mesons in a bin of size $\Delta Y$. The reconstruction acceptance $\mathcal{A}$ takes into account small admixtures in the reconstructed signals from other decay modes, migrations, efficiencies and QED radiative effects for that bin, $\mathcal{L}$ is the integrated luminosity and $\mathcal{B}$ is the branching ratio [41] for the decay channel used in the reconstruction (see Table 1). The total production cross sections were determined using

$$
\sigma=\frac{N(D)}{\mathcal{A} \cdot \mathcal{L} \cdot \mathcal{B}}
$$

where $N(D)$ and $\mathcal{A}$ are now for the whole kinematical range of the measurement.

The reconstruction acceptances were calculated with RAPGAP and vary depending on the particle and the kinematic region of the measurement. For $1.5<Q^{2}<1000 \mathrm{GeV}^{2}, 0.02<$ $y<0.7$, transverse momenta $p_{T}\left(D^{0}, D^{+}\right)>3 \mathrm{GeV}, p_{T}\left(D_{s}^{+}\right)>2 \mathrm{GeV}$ and pseudorapidity $|\eta(D)|<1.6$ the overall acceptances were $\approx 42 \%, \approx 26 \%$ and $\approx 17 \%$ for $D^{0}, D^{+}$, and $D_{s}^{+}$ mesons, respectively.

The relative $b$-quark contributions, predicted by the $\mathrm{MC}$ simulation using branching ratios of $b$-quark decays to the charmed hadrons measured at LEP $[49,50]$, were subtracted from all measured cross sections. The subtraction of the $b$-quark contribution reduced the measured cross sections by $3.1 \%$ for the $D^{0}$ and $D^{+}$and $4.3 \%$ for the $D_{s}^{+}$and changed the measured charm fragmentation ratios and fractions by less than $1 \%$. 


\section{Charm-meson production cross sections}

Charm-meson cross sections were calculated using the reconstructed signals for the process $e p \rightarrow e D X$ in the kinematic region $1.5<Q^{2}<1000 \mathrm{GeV}^{2}, 0.02<y<0.7, p_{T}(D)>3 \mathrm{GeV}$ (for the $D_{s}^{+}$also $p_{T}\left(D_{s}^{+}\right)>2 \mathrm{GeV}$ ) and $|\eta(D)|<1.6$.

The systematic uncertainties presented in this and the following sections will be discussed in Section 11. The third set of uncertainties quoted for the measured cross sections and charm fragmentation ratios and fractions are due to the propagation of the relevant branching-ratio uncertainties.

The following cross sections were measured:

- the production cross section for $D^{0}$ mesons not originating from the $D^{*+} \rightarrow D^{0} \pi_{s}^{+}$ decays, hereafter called untagged $D^{0}$ mesons, is:

$$
\left.\sigma^{\text {untag }}\left(D^{0}\right)=5.56 \pm 0.35 \text { (stat.) }\right)_{-0.26}^{+0.32} \text { (syst.) } \pm 0.10 \text { (br.) nb; }
$$

- the production cross section for $D^{0}$ mesons originating from the $D^{*+} \rightarrow D^{0} \pi_{s}^{+}$decays:

$$
\left.\sigma^{\operatorname{tag}}\left(D^{0}\right)=1.78 \pm 0.08 \text { (stat. }\right)_{-0.10}^{+0.12} \text { (syst.) } \pm 0.03 \text { (br.) nb; }
$$

- the production cross section for all $D^{0}$ mesons:

$$
\left.\sigma\left(D^{0}\right)=\sigma^{\text {untag }}\left(D^{0}\right)+\sigma^{\operatorname{tag}}\left(D^{0}\right)=7.34 \pm 0.36 \text { (stat. }\right)_{-0.27}^{+0.35} \text { (syst.) } \pm 0.13 \text { (br.) nb; }
$$

- the production cross section for additional $D^{*+}$ mesons:

$$
\left.\left.\sigma^{\text {add }}\left(D^{*+}\right)=0.518 \pm 0.046 \text { (stat. }\right)_{-0.046}^{+0.051} \text { (syst. }\right) \pm 0.01 \text { (br.) nb. }
$$

The production cross section for $D^{*+}$ mesons in the kinematic range $p_{T}\left(D^{*+}\right)>3 \mathrm{GeV}$ and $\left|\eta\left(D^{*+}\right)\right|<1.6, \sigma^{\text {kin }}\left(D^{*+}\right)$, is given by the sum $\sigma^{\text {add }}\left(D^{*+}\right)+\sigma^{\operatorname{tag}}\left(D^{0}\right) / \mathcal{B}_{D^{*+} \rightarrow D^{0} \pi^{+}}$:

$$
\left.\sigma^{\text {kin }}\left(D^{*+}\right)=3.14 \pm 0.12 \text { (stat. }\right)_{-0.15}^{+0.18}(\text { syst. }) \pm 0.06 \text { (br.) nb; }
$$

- the production cross section for $D^{+}$mesons:

$$
\left.\sigma\left(D^{+}\right)=2.80 \pm 0.30(\text { stat. })_{-0.14}^{+0.18} \text { (syst. }\right) \pm 0.10(\text { br. }) \mathrm{nb}
$$


- the production cross section for $D_{s}^{+}$mesons with $p_{T}\left(D_{s}^{+}\right)>3 \mathrm{GeV}$ :

$$
\sigma\left(D_{s}^{+}\right)=1.27 \pm 0.16(\text { stat. })_{-0.06}^{+0.11}(\text { syst. })_{-0.15}^{+0.19}(\text { br. }) \mathrm{nb}
$$

- the production cross section for $D_{s}^{+}$mesons with $p_{T}\left(D_{s}^{+}\right)>2 \mathrm{GeV}$ :

$$
\left.\sigma_{2}\left(D_{s}^{+}\right)=2.42 \pm 0.30 \text { (stat. }\right)_{-0.14}^{+0.30}(\text { syst. })_{-0.27}^{+0.35}(\text { br. }) \mathrm{nb} .
$$

\section{Charm fragmentation ratios}

In this section, the ratio of neutral to charged $D$-meson production rates, $R_{u / d}$, the strangeness-suppression factor, $\gamma_{s}$, and the fraction of charged $D$ mesons produced in a vector state, $P_{\mathrm{v}}^{d}$, are presented in the kinematic range $1.5<Q^{2}<1000 \mathrm{GeV}^{2}, 0.02<$ $y<0.7, p_{T}(D)>3 \mathrm{GeV}$ and $|\eta(D)|<1.6$.

\subsection{Ratio of neutral to charged $D$-meson production rates}

Neglecting influences from decays of heavier excited $D$ mesons, the ratio of neutral to charged $D$-meson production rates is given by the ratio of the sum of $D^{* 0}$ and direct $D^{0}$ production cross sections to the sum of $D^{*+}$ and direct $D^{+}$production cross sections. This ratio can be calculated as [2]

$$
R_{u / d}=\frac{\sigma^{\mathrm{untag}}\left(D^{0}\right)}{\sigma\left(D^{+}\right)+\sigma^{\operatorname{tag}}\left(D^{0}\right)}
$$

Using the measured cross sections, the ratio of neutral to charged $D$-meson production rates is

$$
\left.R_{u / d}=1.22 \pm 0.11 \text { (stat. }\right)_{-0.02}^{+0.05} \text { (syst.) } \pm 0.03(\text { br. })
$$

The measured $R_{u / d}$ value agrees with unity, i.e. it is consistent with isospin invariance, which implies that $u$ and $d$ quarks are produced equally in charm fragmentation.

In Table 2 and Fig. 5, this measurement is compared with those obtained in DIS by the H1 collaboration [4], in photoproduction [2] and in $e^{+} e^{-}$annihilations [51]. All measurements agree within experimental uncertainties. 


\subsection{Strangeness-suppression factor}

The strangeness-suppression factor for charm mesons is given by the ratio of twice the production rate of charm-strange mesons to the production rate of non-strange charm mesons. All $D^{*+}$ and $D^{* 0}$ decays produce either a $D^{+}$or a $D^{0}$ meson, while all $D_{s}^{*+}$ decays produce a $D_{s}^{+}$meson [41]. Thus, neglecting decays of heavier excited charmstrange mesons to non-strange charm mesons, the strangeness-suppression factor can be calculated as the ratio of twice the $D_{s}^{+}$cross section to the sum of $D^{0}$ and $D^{+}$cross sections.

For the comparison of the inclusive $D^{+}, D^{0}$ and $D^{*+}$ cross sections with each other, the equivalent phase-space treatment [2] was used. The equivalent $D^{+}$and $D^{0}$ cross sections were defined as the sum of their direct cross sections, i.e. $D^{+}$and $D^{0}$ not coming from $D^{*}$, and the contribution from $D^{*+}$ and $D^{* 0}$ decays [2]:

$$
\begin{aligned}
\sigma^{\mathrm{eq}}\left(D^{+}\right) & =\sigma\left(D^{+}\right)+\sigma^{\text {add }}\left(D^{*+}\right) \cdot\left(1-\mathcal{B}_{D^{*+} \rightarrow D^{0} \pi^{+}}\right), \\
\sigma^{\mathrm{eq}}\left(D^{0}\right) & =\sigma^{\text {untag }}\left(D^{0}\right)+\sigma^{\operatorname{tag}}\left(D^{0}\right)+\sigma^{\text {add }}\left(D^{*+}\right) \cdot \mathcal{B}_{D^{*+} \rightarrow D^{0} \pi^{+}}+\sigma^{\text {add }}\left(D^{* 0}\right) .
\end{aligned}
$$

It was also assumed that the production cross section for "additional" $D^{* 0}$ mesons, producing $D^{0}$ mesons outside of the nominal kinematic range, is $\sigma^{\text {add }}\left(D^{* 0}\right)=R_{u / d} \sigma^{\text {add }}\left(D^{*+}\right)$. The strangeness-suppression factor is then given by

$$
\gamma_{s}=\frac{2 \sigma\left(D_{s}^{+}\right)}{\sigma^{\mathrm{eq}}\left(D^{+}\right)+\sigma^{\mathrm{eq}}\left(D^{0}\right)}=\frac{2 \sigma\left(D_{s}^{+}\right)}{\sigma\left(D^{+}\right)+\sigma^{\operatorname{untag}}\left(D^{0}\right)+\sigma^{\operatorname{tag}}\left(D^{0}\right)+\sigma^{\operatorname{add}}\left(D^{*+}\right) \cdot\left(1+R_{u / d}\right)} .
$$

Using the measured cross sections, the strangeness-suppression factor is

$$
\left.\left.\gamma_{s}=0.225 \pm 0.030 \text { (stat. }\right)_{-0.007}^{+0.018} \text { (syst. }\right)_{-0.026}^{+0.034} \text { (br.). }
$$

Table 3 and Fig. 5 compare this measurement with the values measured in photoproduction [2], in DIS by the H1 collaboration [4] and in $e^{+} e^{-}$annihilations [51]. All measurements agree within experimental uncertainties. The large branching-ratio uncertainties are dominated by the uncertainties of the $D_{s}^{+} \rightarrow \phi \pi^{+}$branching ratio.

\subsection{Fraction of charged $D$ mesons produced in a vector state}

Neglecting influences from decays of heavier excited $D$ mesons, the fraction of charged $D$ mesons produced in a vector state, $P_{\mathrm{v}}^{d}$, is given by the ratio of vector to (vector + pseudoscalar) charm meson production cross sections. The following relation holds [2]: 


$$
P_{\mathrm{v}}^{d}=\frac{\sigma^{\operatorname{tag}}\left(D^{0}\right) / \mathcal{B}_{D^{*+} \rightarrow D^{0} \pi^{+}}+\sigma^{\mathrm{add}}\left(D^{*+}\right)}{\sigma\left(D^{+}\right)+\sigma^{\operatorname{tag}}\left(D^{0}\right)+\sigma^{\operatorname{add}}\left(D^{*+}\right)}
$$

Using the measured cross sections, the fraction of charged $D$ mesons produced in a vector state is

$$
P_{\mathrm{v}}^{d}=0.617 \pm 0.038(\text { stat. })_{-0.009}^{+0.017}(\text { syst. }) \pm 0.017(\text { br. })
$$

The measured $P_{\mathrm{v}}^{d}$ value is smaller than the naive spin-counting prediction of 0.75 . Table 4 and Fig. 5 compare this measurement with the values measured in photoproduction [2], in DIS by the $\mathrm{H} 1$ collaboration [4] and in $e^{+} e^{-}$annihilations [51]. All the measurements are consistent.

\section{Charm fragmentation fractions}

The fraction of $c$ quarks hadronising as a particular charm meson, $f(c \rightarrow D)$, is given by the ratio of the production cross section for the meson to the sum of the production cross sections for all charm ground states that decay weakly, $\sigma_{\text {gs }}$. In addition to the measured $D^{0}, D^{+}$and $D_{s}^{+}$charm ground states, the production cross sections of the $\Lambda_{c}^{+}$baryon and of the charm-strange baryons $\Xi_{c}^{+}, \Xi_{c}^{0}$ and $\Omega_{c}^{0}$ should be included in the sum. The production rates for the latter are expected to be much lower than that of the $\Lambda_{c}^{+}$due to strangeness suppression.

The $\sigma\left(\Lambda_{c}^{+}\right)$was estimated using the corresponding fragmentation fraction measured in $e^{+} e^{-}[51], f\left(c \rightarrow \Lambda_{c}^{+}\right) e_{e^{+} e^{-}}$, by the relation below:

$$
f\left(c \rightarrow \Lambda_{c}^{+}\right) e^{+} e^{-}=\sigma\left(\Lambda_{c}^{+}\right) / \sigma_{\mathrm{gs}}
$$

The uncertainty of this procedure was estimated by using $f\left(c \rightarrow \Lambda_{c}^{+}\right)$obtained in photoproduction [2], and considering the uncertainty in $f\left(c \rightarrow \Lambda_{c}^{+}\right)_{e^{+} e^{-}}[51]$.

The relative rates for the weakly-decaying charm-strange baryons were estimated from the non-charm sector following the LEP procedure [52]. The measured $\Xi^{-} / \Lambda$ and $\Omega^{-} / \Lambda$ relative rates are $(6.65 \pm 0.28) \%$ and $(0.42 \pm 0.07) \%$, respectively [41]. Assuming equal production of $\Xi^{0}$ and $\Xi^{-}$states and that a similar suppression is applicable to the charm baryons, the total rate for the three charm-strange baryons relative to the $\Lambda_{c}^{+}$state is expected to be about $14 \%$. Therefore, the estimated $\Lambda_{c}^{+}$production cross section was scaled by a factor 1.14 in the sum of the production cross sections. An error of \pm 0.05 was assigned to the scale factor when evaluating systematic uncertainties. 
Using the equivalent $D^{0}$ and $D^{+}$cross sections [2], $\sigma_{\mathrm{gs}}$ is given by

$$
\sigma_{\mathrm{gs}}=\sigma^{\mathrm{eq}}\left(D^{+}\right)+\sigma^{\mathrm{eq}}\left(D^{0}\right)+\sigma\left(D_{s}^{+}\right)+\sigma\left(\Lambda_{c}^{+}\right) \cdot 1.14,
$$

which can be expressed as

$$
\sigma_{\mathrm{gs}}=\sigma\left(D^{+}\right)+\sigma^{\mathrm{untag}}\left(D^{0}\right)+\sigma^{\mathrm{tag}}\left(D^{0}\right)+\sigma^{\mathrm{add}}\left(D^{*+}\right) \cdot\left(1+R_{u / d}\right)+\sigma\left(D_{s}^{+}\right)+\sigma\left(\Lambda_{c}^{+}\right) \cdot 1.14
$$

Using the measured cross sections and combining Eqs. (3) and (2) yields

$$
\sigma_{\mathrm{gs}}=13.7 \pm 0.6(\text { stat. })_{-0.6}^{+1.4} \text { (syst.) } \pm 0.6 \text { (br.) nb. }
$$

The fragmentation fractions for the measured charm ground state are given by

$$
\begin{aligned}
f\left(c \rightarrow D^{+}\right) & =\sigma^{\mathrm{eq}}\left(D^{+}\right) / \sigma_{\mathrm{gs}}=\left[\sigma\left(D^{+}\right)+\sigma^{\mathrm{add}}\left(D^{*+}\right) \cdot\left(1-\mathcal{B}_{D^{*+} \rightarrow D^{0} \pi^{+}}\right)\right] / \sigma_{\mathrm{gs}}, \\
f\left(c \rightarrow D^{0}\right) & =\sigma^{\mathrm{eq}}\left(D^{0}\right) / \sigma_{\mathrm{gs}} \\
& =\left[\sigma^{\mathrm{untag}}\left(D^{0}\right)+\sigma^{\mathrm{tag}}\left(D^{0}\right)+\sigma^{\mathrm{add}}\left(D^{*+}\right) \cdot\left(R_{u / d}+\mathcal{B}_{D^{*+} \rightarrow D^{0} \pi^{+}}\right)\right] / \sigma_{\mathrm{gs}}, \\
f\left(c \rightarrow D_{s}^{+}\right) & =\sigma\left(D_{s}^{+}\right) / \sigma_{\mathrm{gs}} .
\end{aligned}
$$

The fragmentation fraction for the $D^{*+}$ is

$$
f\left(c \rightarrow D^{*+}\right)=\sigma^{\mathrm{kin}}\left(D^{*+}\right) / \sigma_{\mathrm{gs}}=\left[\sigma^{\operatorname{tag}}\left(D^{0}\right) / \mathcal{B}_{D^{*+} \rightarrow D^{0} \pi^{+}}+\sigma^{\text {add }}\left(D^{*+}\right)\right] / \sigma_{\mathrm{gs}} .
$$

The open-charm fragmentation fractions, measured in the kinematic region $1.5<Q^{2}<$ $1000 \mathrm{GeV}^{2}, 0.02<y<0.7, p_{T}(D)>3 \mathrm{GeV}$ and $|\eta(D)|<1.6$, are summarised in Table 5 and Fig. 5. The results are compared with the values obtained in photoproduction [2], in DIS by the $\mathrm{H} 1$ collaboration [4] and in $e^{+} e^{-}$annihilations [51]. All the measurements are consistent. A Monte Carlo study [2] suggested that the measured charm fragmentation ratios and fractions are close to those in the full $p_{T}(D)$ and $\eta(D)$ phase space.

The hadronisation fraction into untagged $D^{0}$, needed in the next section for comparisons with theory, was:

$$
\begin{aligned}
f^{\text {untag }}\left(c \rightarrow D^{0}\right) & =f\left(c \rightarrow D^{0}\right)-f\left(c \rightarrow D^{*+}\right) \mathcal{B}_{D^{*+} \rightarrow D^{0} \pi^{+}} \\
& =\left(\sigma^{\text {untag }}\left(D^{0}\right)+\sigma^{\text {add }}\left(D^{* 0}\right)\right) / \sigma_{g s} \\
& \left.\left.=0.450 \pm 0.020 \text { (stat. })_{-0.039}^{+0.009} \text { (syst. }\right)_{-0.017}^{+0.012} \text { (br. }\right)
\end{aligned}
$$

where the equivalent phase-space treatment and $\sigma^{\text {add }}\left(D^{* 0}\right)$ were considered as in Section 8.2. 


\section{Cross sections and pQCD comparisons}

For the cross sections presented in Section 7 the predictions from the HVQDIS program are $\sigma\left(D^{0}\right)=7.90 \mathrm{nb}, \sigma^{\text {untag }}\left(D^{0}\right)=5.88 \mathrm{nb}, \sigma\left(D^{+}\right)=2.82 \mathrm{nb}$ and $\sigma_{2}\left(D_{s}^{+}\right)=2.40 \mathrm{nb}$, with uncertainties around $15 \%$, dominated by the input PDF and the mass of the charm quark. They are in good agreement with the data.

The differential cross sections for untagged $D^{0}$ (the $D^{0}$ mesons coming from $D^{*+}$ are already included in the previous ZEUS publication [3]), $D^{+}$and $D_{s}^{+}$as a function of $Q^{2}$, $x, p_{T}(D)$ and $\eta(D)$ are shown in Figs. 6, 7 and 8 and given in Tables 6 and 7 . The cross sections in $Q^{2}$ and $x$ both fall by about three orders of magnitude in the measured region. The cross-section $d \sigma / d p_{T}(D)$ falls by two orders of magnitude with increasing $p_{T}(D)$. The cross-section $d \sigma / d \eta\left(D^{0}\right)$ shows a mild increase with increasing $\eta\left(D^{0}\right)$; for the $D^{+}$and $D_{s}^{+}$ no statistically significant dependence with $\eta(D)$ is observed.

Figures 6, 7 and 8 show also the corresponding NLO calculations implemented in the HVQDIS program as well as their uncertainties (Section 11.2). All the differential cross sections measured are well described by the NLO calculation.

\section{Systematic uncertainties}

\subsection{Systematic uncertainties of measurements}

The systematic uncertainties of the measured cross sections and fragmentation ratios and fractions were determined by changing the analysis procedure and repeating all calculations.

In the measurement of fragmentation ratios and fractions the following groups of systematic uncertainty sources were considered (Table 8):

- $\left\{\delta_{1}\right\}$ the model dependence of the acceptance corrections was estimated using the HeRwig MC sample;

- $\left\{\delta_{2}\right\}$ the uncertainty of the beauty subtraction was determined by varying the $b$-quark cross section by a factor of two in the reference MC sample;

- $\left\{\delta_{3}\right\}$ the uncertainty of the tracking simulation was obtained by varying all momenta by $\pm 0.3 \%$ which corresponds to the uncertainty in the magnetic field; and by changing the track momentum resolution and the angular resolution by ${ }_{-10}^{+20 \%}$ of their values. The asymmetric resolution variations were used since the MC signals typically had somewhat narrower widths than observed in the data; 
- $\left\{\delta_{4}\right\}$ the uncertainty in the CAL energy scale was studied by varying in the MC the energy of the scattered $e^{-}$by $\pm 1 \%$ and the energy of the hadronic system by $\pm 3 \%$;

- $\left\{\delta_{5}\right\}$ the uncertainties related to the signal extraction procedures were studied as follows:

- the cuts on the minimum $p_{T}$ for the $\pi$ and $K$ candidates were independently raised and lowered by $10 \%$ from their nominal values,

- the cut on the minimum $p_{T}$ for the $\pi_{s}$ was raised and lowered by $0.02 \mathrm{GeV}$ (for $\left.\sigma^{\operatorname{tag}}\left(D^{0}\right), \sigma^{\text {untag }}\left(D^{0}\right), \sigma^{\text {add }}\left(D^{*+}\right)\right)$,

- the $\Delta M$ signal region was widened symmetrically by $0.003 \mathrm{GeV}$ (for $\sigma^{\operatorname{tag}}\left(D^{0}\right)$, $\left.\sigma^{\text {untag }}\left(D^{0}\right), \sigma^{\text {add }}\left(D^{*+}\right)\right)$,

- the $M(K \pi)$ signal region was widened and narrowed symmetrically by $0.01 \mathrm{GeV}$ (for $\left.\sigma^{\text {add }}\left(D^{*+}\right)\right)$,

- the wrong-charge background normalisation region was changed to $0.152<\Delta M<$ 0.168 (for $\left.\sigma^{\text {add }}\left(D^{*+}\right)\right)$;

- $\left\{\delta_{6}\right\}$ the uncertainties of the luminosities of the $e^{-} p( \pm 1.8 \%)$ and $e^{+} p( \pm 2.25 \%)$ data samples were included, taking into account their correlations;

- $\left\{\delta_{7}\right\}$ the uncertainty in the estimate of $\sigma\left(\Lambda_{c}^{+}\right)$(see Section 9);

- $\left\{\delta_{8}\right\}$ the uncertainty in the rate of the charm-strange baryons (see Section 9);

Contributions from the different systematic uncertainties were calculated and added in quadrature separately for positive and negative variations. Correlated systematic uncertainties largely cancel in the calculation of the fragmentation ratios and fractions.

For the total and differential cross-section measurements discussed in Section 10 and those used for the extraction of $F_{2}^{c \bar{c}}$ (Section 12), further sources of systematics were studied $[3,5,53],\left\{\delta_{9}\right\}$ :

- the cut on $y_{e}$ was changed to $y_{e} \leq 0.90$;

- the cut on $y_{\mathrm{JB}}$ was changed to $y_{\mathrm{JB}} \geq 0.03$;

- the cut on $\delta$ was changed to $42 \leq \delta \leq 70 \mathrm{GeV}$;

- the cut on $\left|Z_{\text {vertex }}\right|$ was changed to $\left|Z_{\text {vertex }}\right|<45 \mathrm{~cm}$;

- the cut on $E_{e^{\prime}}$ was changed to $E_{e^{\prime}}>11 \mathrm{GeV}$;

- the excluded region for the impact position of the scattered electron in the RCAL was increased by $1 \mathrm{~cm}$ in each direction;

- the electron method was used, except for cases when the scattered electron track was reconstructed by the CTD. In the latter case, the DA method, which has the best resolution at high $Q^{2}$, was used. 
These estimations were made in each bin in which the differential cross sections were measured. In addition, for the lowest $x$ bin of the differential cross section of untagged $D^{0}$, the systematic error accounted also for instabilities in the signal extraction, not encountered in any other bin. The overall systematic uncertainty was determined by adding the individual uncertainties in quadrature. Typically $\delta_{9}$ was below $4 \%$. The uncertainty on the luminosity measurement was not included in the systematic uncertainties for the differential cross sections.

\subsection{Uncertainties on theoretical predictions}

The NLO QCD predictions for $D$ meson production are affected by the systematic uncertainties listed below. Typical values are quoted for the total cross section:

- the ZEUS PDF uncertainties were propagated from the experimental uncertainties of the fitted data $( \pm 5 \%)$. As an alternative parametrisation in the FFNS, the CTEQ5F3 PDF was used in HVQDIS with a charm mass of $1.3 \mathrm{GeV}(-2 \%)$;

- the charm mass was changed simultaneously in the PDF fit and in HVQDIS by $\mp 0.15 \mathrm{GeV}\left({ }_{-8}^{+8} \%\right)$. The largest effect was at low $p_{T}(D)$;

- the scale was changed to $2 \sqrt{Q^{2}+4 m_{c}^{2}}$ and to $\max \left(\sqrt{Q^{2} / 4+m_{c}^{2}}, 2 m_{c}\right)\left({ }_{-6}^{+5} \%\right)$;

- the JETSET fragmentation as implemented in the previous analyses $[3,5]$ was used instead of the Peterson fragmentation $(+5 \%$ to $+20 \%)$. The largest deviations were observed for $D^{0}$ and $D^{+}$particles at the lowest $Q^{2}$ and $x$.

\section{Extraction of $F_{2}^{c \bar{c}}$}

The open charm contribution, $F_{2}^{c \bar{c}}$, to the proton structure-function $F_{2}$ can be defined in terms of the inclusive double-differential $c \bar{c}$ cross section in $x$ and $Q^{2}$ by

$$
\frac{d^{2} \sigma^{c \bar{c}}\left(x, Q^{2}\right)}{d x d Q^{2}}=\frac{2 \pi \alpha^{2}}{x Q^{4}}\left\{\left[1+(1-y)^{2}\right] F_{2}^{c \bar{c}}\left(x, Q^{2}\right)-y^{2} F_{L}^{c \bar{c}}\left(x, Q^{2}\right)\right\}
$$

In this paper, the $c \bar{c}$ cross section is obtained by measuring the untagged $D^{0}, D^{+}$and $D_{s}^{+}$ production cross sections and employing the measured hadronisation fractions $f(c \rightarrow D)$. Since only a limited kinematic region in $p_{T}(D)$ and $\eta(D)$ is accessible, a prescription for extrapolating to the full kinematic phase space is needed.

As reported in Section 10, the measured differential cross-sections are well described in the probed kinematic region. Therefore the following relation was used to extract $F_{2}^{c \bar{c}}$ : 


$$
F_{2}^{c \bar{c}}\left(x_{i}, Q_{i}^{2}\right)=\frac{\sigma_{i, \text { meas }}(e p \rightarrow D X)}{\sigma_{i, \text { theo }}(e p \rightarrow D X)} F_{2, \text { theo }}^{c \bar{c}}\left(x_{i}, Q_{i}^{2}\right)
$$

where $\sigma_{i \text {,meas }}$ is the cross section in the bin $i$ in the measured region of $p_{T}(D)$ and $\eta(D)$ and $\sigma_{i, \text { theo }}$ is the corresponding cross section evaluated with HVQDIS. The value of $F_{2}^{c \bar{c}}$ theo was calculated in FFNS from the NLO coefficient functions [27] using the same values of parameters as in the calculation of $\sigma_{i, \text { theo }}$. The cross sections $\sigma_{i, \text { meas }}(e p \rightarrow D X)$ were measured in bins of $Q^{2}$ and $y$ (Table 9) and $F_{2}^{c \bar{c}}$ was quoted at representative $Q^{2}$ and $x$ values for each bin (Table 10). The $F_{2}^{c c}$ measurements obtained from each $D$ meson were combined into a single set of measurements; the result is also shown in Table 10.

The extrapolation factors from the measured $p_{T}(D)$ and $\eta(D)$ ranges to the full phase space, as estimated using HVQDIS, were between 17 at low $Q^{2}$ and 2.5 at high $Q^{2}$ for the $D^{0}$ and $D^{+}$measurements. For the $D_{s}^{+}$, the lower $p_{T}$ requirement leads to lower extrapolation factors between 5.6 and 1.9. They are all shown in Table 10. The uncertainty from the branching ratios was estimated by changing each branching ratio independently in the calculation by \pm 1 standard deviation and adding in quadrature the resulting variations of $F_{2}^{c \bar{c}}\left({ }_{-4.1}^{+2.7 \%)}\right.$.

The following uncertainties of the extrapolation prescription of Eq. (4) have been evaluated:

- using Jetset instead of the Peterson fragmentation yielded changes of $\approx+28 \%,+15 \%$ and $+5 \%$ for the data points at the lowest, middle and largest $Q^{2}$ ranges, respectively;

- changing the charm mass by $\pm 0.15 \mathrm{GeV}$ consistently in the HVQDIS calculation and in the calculation of $F_{2 \text {,theo }}^{c \bar{c}}$ led to differences in the extrapolation of $\pm 5 \%$ at low $x$, low $Q^{2}$; the value decreases rapidly at higher $x$ and higher $Q^{2}$;

- the uncertainty in the ZEUS NLO PDF fit led to uncertainties in the extracted values of $F_{2}^{c \bar{c}}$ typically less than $1 \%$;

- the extrapolation factors were evaluated using the CTEQ5F3 proton PDF yielding differences compared to the nominal factors of $\approx+10 \%,+6 \%$ and $+1 \%$ for the lowest, middle and largest $Q^{2}$ ranges, respectively.

The combined $F_{2}^{c \bar{c}}$ measurements are shown in Fig. 9. The quadratic addition of the extrapolation uncertainties is shown independently as a band. Also shown in Fig. 9 is the previous measurement [3] and the ZEUS NLO QCD fit. The two sets of data are consistent $^{3}$. The prediction describes the data well for all $Q^{2}$ and $x$. The uncertainty

\footnotetext{
${ }^{3}$ The previous data were measured at $Q^{2}=4,18$ and $130 \mathrm{GeV}^{2}$ and have been shifted to $Q^{2}=4.2$, 20.4 and $111.8 \mathrm{GeV}^{2}$ using the ZEUS NLO QCD fit.
} 
on the theoretical prediction is that from the PDF fit propagated from the experimental uncertainties of the fitted data.

\section{Summary and conclusions}

The production of the charm mesons $D^{*+}, D^{0}, D^{+}$and $D_{s}^{+}$has been measured with the ZEUS detector in the kinematic range $1.5<Q^{2}<1000 \mathrm{GeV}^{2}, 0.02<y<0.7$, $p_{T}\left(D^{*+}, D^{0}, D^{+}\right)>3 \mathrm{GeV}, p_{T}\left(D_{s}^{+}\right)>2 \mathrm{GeV}$ and $|\eta(D)|<1.6$.

The cross sections have been used to determine the charm fragmentation ratios and fractions. The ratio of neutral to charged $D$-meson production, $R_{u / d}$, is compatible with unity, i.e. it is consistent with isospin invariance, which implies that $u$ and $d$ quarks are produced equally in charm fragmentation. The strangeness-suppression factor in charm fragmentation, $\gamma_{s}$, was measured to be about $20 \%$. The fraction of charged $D$ mesons produced in a vector state, $P_{\mathrm{v}}^{d}$, was found to be smaller than the naive spin-counting prediction of 0.75 . The fraction of $c$ quarks hadronising as $D^{*+}, D^{0}, D^{+}$and $D_{s}^{+}$mesons have been calculated. The measured $R_{u / d}, \gamma_{s}, P_{\mathrm{v}}^{d}$ and open charm fragmentation fractions are consistent with those obtained in charm photoproduction and in $e^{+} e^{-}$annihilation. These measurements generally support the hypothesis that fragmentation proceeds independently of the hard sub-process.

The measured $D^{0}, D^{+}$and $D_{s}^{+}$differential cross sections were compared to the predictions of NLO QCD with the proton PDFs extracted from inclusive DIS data. A good description was found.

The double-differential cross section in $y$ and $Q^{2}$ has been used to extract the open charm contribution to $F_{2}$, by using the NLO QCD calculation to extrapolate outside the measured $p_{T}(D)$ and $\eta(D)$ regions. The $F_{2}^{c \bar{c}}$ values obtained from the different $D$ mesons agree with previous results where a $D^{*+}$ meson was tagged.

\section{Acknowledgments}

The strong support and encouragement of the DESY Directorate have been invaluable, and we are much indebted to the HERA machine group for their inventiveness and diligent efforts. The design, construction and installation of the ZEUS detector have been made possible by the ingenuity and dedicated efforts of many people from inside DESY and from the home institutes who are not listed as authors. Their contributions are acknowledged with great appreciation. 


\section{References}

[1] For a review see J.C. Collins, D.E. Soper and G. Sterman, Factorization of Hard Process in QCD, in "Perturbative Quantum Chromodynamics", A.H. Mueller (ed.). World Scientific, Singapore, 1989.

[2] ZEUS Coll., S. Chekanov et al., Eur. Phys. J. C 44 (2005) 351.

[3] ZEUS Coll., S. Chekanov et al., Phys. Rev. D 69 (2004) 012004.

[4] H1 Coll., A. Aktas et al., Eur. Phys. J. C 38 (2005) 447.

[5] ZEUS Coll., J. Breitweg et al., Eur. Phys. J. C 12 (2000) 35.

[6] ZEUS Coll., J. Breitweg et al., Phys. Lett. B 407 (1997) 402.

[7] H1 Coll., C. Adloff et al., Phys. Lett. B 528 (2002) 199.

[8] H1 Coll., C. Adloff et al., Z. Phys. C 72 (1996) 593.

[9] H1 Coll., C. Adloff et al., Nucl. Phys. B 545 (1999) 21.

[10] H1 Coll., A. Aktas et al., Eur. Phys. J. C 40 (2005) 349.

[11] ZEUS Coll., J. Breitweg et al., Phys. Lett. B 481 (2000) 213.

[12] ZEUS Coll., J. Breitweg et al., Eur. Phys. J. C 6 (1999) 67.

[13] ZEUS Coll., J. Breitweg et al., Phys. Lett. B 401 (1997) 192.

[14] H1 Coll., A. Aktas et al., Phys. Lett. B 621 (2005) 56.

[15] H1 Coll., S. Aid et al., Nucl. Phys. B 472 (1996) 32.

[16] ZEUS Coll., U. Holm (ed.), The ZEUS Detector. Status Report (unpublished), DESY (1993), available on http://www-zeus.desy.de/bluebook/bluebook.html.

[17] N. Harnew et al., Nucl. Instr. Meth. A 279 (1989) 290;

B. Foster et al., Nucl. Phys. Proc. Suppl. B 32 (1993) 181;

B. Foster et al., Nucl. Instr. Meth. A 338 (1994) 254.

[18] M. Derrick et al., Nucl. Instr. Meth. A 309 (1991) 77;

A. Andresen et al., Nucl. Instr. Meth. A 309 (1991) 101;

A. Caldwell et al., Nucl. Instr. Meth. A 321 (1992) 356;

A. Bernstein et al., Nucl. Instr. Meth. A 336 (1993) 23.

[19] A. Bamberger et al., Nucl. Instr. Meth. A 401 (1997) 63.

[20] A. Dwurazny et al., Nucl. Instr. Meth. A 277 (1989) 176.

[21] J. Andruszków et al., Preprint DESY-92-066, DESY, 1992; ZEUS Coll., M. Derrick et al., Z. Phys. C 63 (1994) 391; J. Andruszków et al., Acta Phys. Pol. B 32 (2001) 2025. 
[22] B.W. Harris and J. Smith, Phys. Rev. D 57 (1998) 2806.

[23] V.N. Gribov and L.N. Lipatov, Sov. J. Nucl. Phys. 15 (1972) 438;

L.N. Lipatov, Sov. J. Nucl. Phys. 20 (1975) 94;

G. Altarelli and G. Parisi, Nucl. Phys. B 126 (1977) 298;

Yu.L. Dokshitzer, Sov. Phys. JETP 46 (1977) 641.

[24] B.W. Harris and J. Smith, Nucl. Phys. B 452 (1995) 109;

B.W. Harris and J. Smith, Phys. Lett. B 353 (1995) 535. Erratum-ibid B 359 (1995) 423.

[25] E. Laenen et al., Nucl. Phys. B 392 (1993) 162;

E. Laenen et al., Nucl. Phys. B 392 (1993) 229.

[26] A. Chuvakin, J. Smith and B.W. Harris, Eur. Phys. J. C 18 (2001) 547.

[27] ZEUS Coll., S. Chekanov et al., Phys. Rev. D 67 (2003) 012007;

Public access to ZEUS 2002 PDFs, available on http://www-pnp.physics.ox.ac.uk/ cooper/zeus2002.html.

[28] C. Peterson et al., Phys. Rev. D 27 (1983) 105.

[29] P. Nason and C. Oleari, Nucl. Phys. B 565 (2000) 245.

[30] H1 Coll., C. Adloff et al., Eur. Phys. J. C 25 (2002) 41.

[31] ZEUS Coll., J. Breitweg et al., Eur. Phys. J. C 6 (1999) 603.

[32] S. Bentvelsen, J. Engelen and P. Kooijman, Proc. Workshop on Physics at HERA, W. Buchmüller and G. Ingelman (eds.), Vol. 1, p. 23. Hamburg, Germany, DESY (1992);

K.C. Höger, ibid., p. 43.

[33] F. Jacquet and A. Blondel, Proceedings of the Study for an ep Facility for Europe, U. Amaldi (ed.), p. 391. Hamburg, Germany (1979). Also in preprint DESY 79/48.

[34] U. Bassler and G. Bernardi, Nucl. Instr. Meth. A 361 (1995) 197.

[35] W.H. Smith, K. Tokushuku and L.W. Wiggers, Proc. Computing in High-Energy Physics (CHEP), Annecy, France, Sept. 1992, C. Verkerk and W. Wojcik (eds.), p. 222. CERN, Geneva, Switzerland (1992). Also in preprint DESY 92-150B.

[36] ZEUS Coll., S. Chekanov et al., Eur. Phys. J. C 21 (2001) 443.

[37] H. Abramowicz, A. Caldwell and R. Sinkus, Nucl. Instr. Meth. A 365 (1995) 508.

[38] R. Sinkus and T. Voss, Nucl. Instr. Meth. A 391 (1997) 360.

[39] G.M. Briskin. Ph.D. Thesis, Tel Aviv University, 1998. DESY-THESIS-1998-036.

[40] D. Bailey and R. Hall-Wilton, Nucl. Instr. Meth. A 515 (2003) 37. 
[41] Particle Data Group, W.-M. Yao et al., J. Phys. G 33 (2006) 1.

[42] H. Jung, Comput. Phys. Comm. 86 (1995) 147.

[43] G. Marchesini et al., Preprint Cavendish-HEP-99/17 (hep-ph/9912396), 1999;

G. Marchesini et al., Comput. Phys. Comm. 67 (1992) 465.

[44] A. Kwiatkowski, H. Spiesberger and H.-J. Möhring, Comput. Phys. Comm. 69 (1992) 155. Also in Proc. Workshop Physics at HERA, 1991, DESY, Hamburg.

[45] R. Brun et al., Geant3, Technical Report CERN-DD/EE/84-1, CERN, 1987.

[46] CTEQ Coll., H.L. Lai et al., Eur. Phys. J. C 12 (2000) 375.

[47] M. Glück, E. Reya and A. Vogt, Phys. Rev. D 46 (1992) 1973.

[48] B.R. Webber, Nucl. Phys. B 238 (1984) 492.

[49] ALEPH Coll., D. Buskulic et al., Phys. Lett. B 388 (1996) 648.

[50] OPAL Coll., K. Ackerstaff et al., Eur. Phys. J. C 1 (1998) 439.

[51] L. Gladilin, Preprint hep-ex/9912064, 1999.

[52] OPAL Coll., G. Alexander et al., Z. Phys. C 72 (1996) 1.

[53] M. Zambrana. Ph.D. Thesis, Universidad Autónoma de Madrid, 2007. (Unpublished).

[54] Particle Data Group, S. Eidelman et al., Phys. Lett. B 592 (2004) 1. 


\begin{tabular}{|c|c|}
\hline Decay mode & Branching ratio \\
\hline \hline$D^{0} \rightarrow K^{-} \pi^{+}$ & $0.0380 \pm 0.0007$ \\
\hline$D^{*+} \rightarrow D^{0} \pi_{s}^{+}$ & $0.6770 \pm 0.0050$ \\
\hline$D^{+} \rightarrow K^{-} \pi^{+} \pi^{+}$ & $0.0951 \pm 0.0034$ \\
\hline$D_{s}^{+} \rightarrow \phi \pi^{+} \rightarrow K^{+} K^{-} \pi^{+}$ & $0.0216 \pm 0.0028$ \\
\hline
\end{tabular}

Table 1: Branching ratios of the D-meson decay modes [41]. 


\begin{tabular}{|c|c|}
\hline & $R_{u / d}$ \\
\hline \hline ZEUS $(\mathrm{DIS})$ & $1.22 \pm 0.11(\text { stat. })_{-0.02}^{+0.05}$ (syst.) $\pm 0.03($ br. $)$ \\
\hline ZEUS $(\gamma p)[2]$ & $1.100 \pm 0.078(\text { stat. })_{-0.061}^{+0.038}(\text { syst. })_{-0.049}^{+0.047}($ br. $)$ \\
\hline combined $e^{+} e^{-}$data & $1.020 \pm 0.069(\text { stat. } \oplus \text { syst. })_{-0.047}^{+0.045}($ br. $)$ \\
\hline H1 (DIS) $[4]$ & $1.26 \pm 0.20$ (stat.) \pm 0.11 (syst.) $\pm 0.04($ br. $\oplus$ theory $)$ \\
\hline
\end{tabular}

Table 2: The ratio of neutral to charged $D$-meson production rates, $R_{u / d}$. The $e^{+} e^{-}$values are taken from [2]; they are an update of the compilation in [51] using the branching-ratio values of [54]. The measurements in this paper, ZEUS (DIS), took the values for all the branching ratios involved from [41], the rest of the quoted measurements took them from [54].

\begin{tabular}{|c|c|}
\hline & $\gamma_{s}$ \\
\hline ZEUS (DIS) & $0.225 \pm 0.030$ (stat. $)_{-0.007}^{+0.018}$ (syst. $)_{-0.026}^{+0.034}$ (br.) \\
\hline ZEUS $(\gamma p)[2]$ & $0.257 \pm 0.024$ (stat.) $)_{-0.016}^{+0.013}$ (syst. $)_{-0.049}^{+0.078}$ (br.) \\
\hline ZEUS $(\gamma p)[11]$ & $0.27 \pm 0.04$ (stat. ${ }_{-0.03}^{+0.02}$ (syst.) \pm 0.07 (br.) \\
\hline combined $e^{+} e^{-}$data & $0.259 \pm 0.023$ (stat. $\oplus$ syst. $)_{-0.052}^{+0.087}$ (br.) \\
\hline H1 (DIS) [4] & $0.36 \pm 0.10$ (stat.) \pm 0.01 (syst.) \pm 0.08 (br. $\oplus$ theory) \\
\hline
\end{tabular}

Table 3: The strangeness-suppression factor in charm fragmentation, $\gamma_{s}$. The $e^{+} e^{-}$values are taken from [2]; they are an update of the compilation in [51] using the branching-ratio values of [54]. The measurements in this paper, ZEUS (DIS), took the values for all the branching ratios involved from [41], the rest of the quoted measurements took them from [54]. 


\begin{tabular}{|c|c|}
\hline & $\overline{P_{\mathrm{v}}^{d}}$ \\
\hline ZEUS (DIS) & 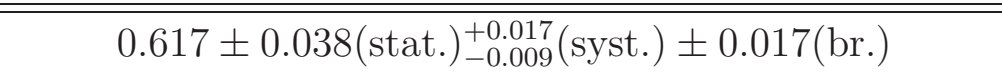 \\
\hline ZEUS $(\gamma p)[2]$ & $0.566 \pm 0.025$ (stat.) ${ }_{-0.022}^{+0.007}$ (syst. ${ }_{-0.023}^{+0.022}$ (br.) \\
\hline combined $e^{+} e^{-}$data & $0.614 \pm 0.019$ (stat. $\oplus$ syst. $)_{-0.025}^{+0.023}$ (br.) \\
\hline H1 (DIS) [4] & $0.693 \pm 0.045$ (stat.) \pm 0.004 (syst.) \pm 0.009 (br. $\oplus$ theory) \\
\hline
\end{tabular}

Table 4: The fraction of charged $D$ mesons produced in a vector state, $P_{\mathrm{v}}^{d}$. The $e^{+} e^{-}$values are taken from [2]; they are an update of the compilation in [51] using the branching-ratio values of [54]. The measurements in this paper, ZEUS (DIS), took the values for all the branching ratios involved from [41], the rest of the quoted measurements took them from [54]. 


\begin{tabular}{|c|c|c|c|c|}
\hline & $\begin{array}{c}\text { ZEUS (DIS) } \\
p_{T}(D)>3 \mathrm{GeV} \\
|\eta(D)|<1.6\end{array}$ & $\begin{array}{c}\text { ZEUS }(\gamma p)[2] \\
p_{T}(D)>3.8 \mathrm{GeV} \\
|\eta(D)|<1.6\end{array}$ & $\begin{array}{c}\text { Combined } \\
e^{+} e^{-} \text {data }[51]\end{array}$ & $\begin{array}{c}\text { H1 (DIS) } \\
{[4]}\end{array}$ \\
\hline & stat. syst. br. & stat. syst. & stat. $\oplus$ syst. br. & total \\
\hline$f\left(c \rightarrow D^{+}\right)$ & $0.216 \pm 0.019_{-0.020-0.010}^{+0.002+0.008}$ & $0.217 \pm 0.014_{-0.005}^{+0.013}$ & $0.226 \pm 0.010{ }_{-0.014}^{+0.016}$ & $0.203 \pm 0.026$ \\
\hline$f\left(c \rightarrow D^{0}\right)$ & $0.605 \pm 0.020_{-0}^{+0 .}$ & $0.523 \pm 0.021_{-0.017}^{+0.018}$ & $0.557 \pm 0.023{ }_{-0.013}^{+0.014}$ & $0.560 \pm 0.046$ \\
\hline$f\left(c \rightarrow D_{s}^{+}\right)$ & $0.092 \pm 0.011_{-0.008}^{+0.007+0.012}$ & $0.095 \pm 0.008_{-0.005}^{+0.005}$ & $0.101 \pm 0.009{ }_{-0.020}^{+0.034}$ & $0.151 \pm 0.055$ \\
\hline$f\left(c \rightarrow D^{*+}\right)$ & $0.229 \pm 0.011_{-0.021-0.010}^{+0.006+007}$ & $0.200 \pm 0.009_{-0.006}^{+0.008}$ & $0.238 \pm 0.007{ }_{-0.003}^{+0.003}$ & $0.263 \pm 0.032$ \\
\hline
\end{tabular}

Table 5: The fractions of c quarks hadronising as a particular charm hadron, $f(c \rightarrow D)$. The fractions are shown for the $D^{+}, D^{0}$ and $D_{s}^{+}$charm ground states and for the $D^{*+}$ state. The measurements in this paper, ZEUS (DIS), took the values for all the branching ratios involved from [41], the rest of the quoted measurements took them from [54]. 


\begin{tabular}{|c|c|c|c|c|c|c|}
\hline & \multicolumn{3}{|c|}{ untagged $D^{0}$} & \multicolumn{3}{|c|}{$D^{+}$} \\
\hline$Q^{2} \operatorname{bin}\left(\mathrm{GeV}^{2}\right)$ & $d \sigma / d Q^{2}$ & $\begin{array}{c}\Delta_{\text {stat }} \\
\left(\mathrm{nb} / \mathrm{GeV}^{2}\right)\end{array}$ & $\Delta_{\text {syst }}$ & $d \sigma / d Q^{2}$ & $\begin{array}{c}\Delta_{\text {stat }} \\
\left(\mathrm{nb} / \mathrm{GeV}^{2}\right)\end{array}$ & $\Delta_{\text {syst }}$ \\
\hline $1.5,5$ & 0.56 & \pm 0.08 & $\begin{array}{l}+0.09 \\
-0.03\end{array}$ & 0.275 & \pm 0.066 & $\begin{array}{l}+0.056 \\
-0.044\end{array}$ \\
\hline 5,15 & 0.141 & \pm 0.008 & ${ }_{-0.006}^{+0.011}$ & 0.089 & \pm 0.014 & ${ }_{-0.010}^{+0.003}$ \\
\hline 15,40 & 0.044 & \pm 0.005 & $\begin{array}{l}+0.003 \\
{ }_{-0.002}\end{array}$ & 0.016 & \pm 0.003 & $\begin{array}{l}+0.002 \\
+0.001\end{array}$ \\
\hline 40,1000 & 0.0012 & \pm 0.0002 & $\begin{array}{l}+0.0002 \\
-0.0001 \\
\end{array}$ & 0.0007 & \pm 0.0002 & $\begin{array}{l}+0.0001 \\
-0.0001 \\
\end{array}$ \\
\hline$x$ bin & $d \sigma / d x$ & $\begin{array}{l}\Delta_{\text {stat }} \\
(\mathrm{nb})\end{array}$ & $\Delta_{\text {syst }}$ & $d \sigma / d x$ & $\begin{array}{l}\Delta_{\text {stat }} \\
(\mathrm{nb})\end{array}$ & $\Delta_{\text {syst }}$ \\
\hline $0.000021,0.0001$ & 15197 & \pm 2543 & $\begin{array}{l}+1137 \\
-5789\end{array}$ & 4470 & \pm 1572 & $\begin{array}{l}{ }_{-1112}^{+1078} \\
\quad\end{array}$ \\
\hline $0.0001,0.0005$ & 4162 & \pm 304 & $\begin{array}{l}+457 \\
-231\end{array}$ & 2295 & \pm 327 & $\begin{array}{l}+157 \\
{ }_{-199}\end{array}$ \\
\hline $0.0005, \quad 0.001$ & 1476 & \pm 195 & ${ }_{-72}^{+128}$ & 1049 & \pm 178 & $\begin{array}{l}+39 \\
-125\end{array}$ \\
\hline $0.001,0.1$ & 20.7 & \pm 2.3 & $\begin{array}{l}+2.7 \\
-1.0 \\
\end{array}$ & 10.5 & \pm 2.2 & $\begin{array}{l}+1.4 \\
-0.5 \\
\end{array}$ \\
\hline$p_{T}(D)$ bin $(\mathrm{GeV})$ & $d \sigma / d p_{T}\left(D^{0}\right)$ & $\begin{array}{c}\Delta_{\text {stat }} \\
(\mathrm{nb} / \mathrm{GeV})\end{array}$ & $\overline{\Delta_{\text {syst }}}$ & $\overline{d \sigma / d p_{T}\left(D^{+}\right)}$ & $\begin{array}{c}\Delta_{\text {stat }} \\
(\mathrm{nb} / \mathrm{GeV})\end{array}$ & $\Delta_{\text {syst }}$ \\
\hline $3.0,3.5$ & 3.13 & \pm 0.39 & $\begin{array}{l}+0.28 \\
-0.12\end{array}$ & 1.61 & \pm 0.44 & $\begin{array}{l}+0.45 \\
-0.21\end{array}$ \\
\hline $3.5,4.5$ & 1.93 & \pm 0.20 & $\begin{array}{l}+0.12 \\
{ }_{-0.11}\end{array}$ & 0.77 & \pm 0.14 & $\begin{array}{l}{ }_{-0.06}^{+0.09} \\
\quad-0.00\end{array}$ \\
\hline $4.5,6.0$ & 0.78 & \pm 0.11 & ${ }_{-0.08}^{+0.05}$ & 0.49 & \pm 0.08 & ${ }_{-0.02}^{+0.05}$ \\
\hline $6.0,20$. & 0.051 & \pm 0.009 & $\begin{array}{l}+0.004 \\
-0.003 \\
\end{array}$ & 0.028 & \pm 0.007 & $\begin{array}{l}+0.002 \\
-0.001 \\
\end{array}$ \\
\hline$\eta(D)$ bin & $d \sigma / d \eta\left(D^{0}\right)$ & $\begin{array}{l}\Delta_{\text {stat }} \\
(\mathrm{nb})\end{array}$ & $\Delta_{\text {syst }}$ & $d \sigma / d \eta\left(D^{+}\right)$ & $\begin{array}{l}\Delta_{\text {stat }} \\
(\mathrm{nb})\end{array}$ & $\Delta_{\text {syst }}$ \\
\hline$-1.6,-0.6$ & 1.18 & \pm 0.19 & $\begin{array}{l}+0.13 \\
-0.10\end{array}$ & 0.65 & \pm 0.11 & $\begin{array}{l}+0.08 \\
{ }_{-0.09}\end{array}$ \\
\hline$-0.6, \quad 0.0$ & 1.59 & \pm 0.19 & $\begin{array}{l}+0.10 \\
-0.11\end{array}$ & 1.20 & \pm 0.25 & $\begin{array}{l}+0.15 \\
-0.22\end{array}$ \\
\hline $0.0, \quad 0.6$ & 2.05 & \pm 0.22 & $\begin{array}{l}+0.18 \\
-0.14\end{array}$ & 1.06 & \pm 0.21 & $\begin{array}{l}+0.08 \\
{ }_{-0.15}\end{array}$ \\
\hline $0.6,1.6$ & 2.31 & \pm 0.37 & $\begin{array}{l}+0.09 \\
-0.20 \\
\end{array}$ & 0.74 & \pm 0.23 & $\begin{array}{l}{ }_{-0.07}^{+0.22} \\
-0.07\end{array}$ \\
\hline
\end{tabular}

Table 6: $\quad$ Measured differential cross sections for $D^{0}$ not coming from a $D^{*+}$ (left), and $D^{+}$(right) as a function of $Q^{2}, x, p_{T}(D)$ and $\eta(D)$ for $1.5<Q^{2}<$ $1000 \mathrm{GeV}^{2}, 0.02<y<0.7, p_{T}(D)>3 \mathrm{GeV}$ and $|\eta(D)|<1.6$. The estimated $b$-quark contribution of $3.1 \%$ has been subtracted. The statistical and systematic uncertainties are shown separately. The $D^{0}\left(D^{+}\right)$cross sections have a further $1.8 \%$ (3.6\%) uncertainty from the $D^{0} \rightarrow K^{-} \pi^{+}\left(D^{+} \rightarrow K^{-} \pi^{+} \pi^{+}\right)$branching ratios. 


\begin{tabular}{|c|c|c|c|}
\hline$Q^{2}$ bin $\left(\mathrm{GeV}^{2}\right)$ & $d \sigma / d Q^{2}$ & $\begin{array}{c}\Delta_{\text {stat }} \\
\left(\mathrm{nb} / \mathrm{GeV}^{2}\right)\end{array}$ & $\Delta_{\text {syst }}$ \\
\hline $1.5,5$ & 0.31 & \pm 0.07 & $\begin{array}{l}+0.08 \\
-0.05\end{array}$ \\
\hline 5,15 & 0.092 & \pm 0.016 & ${ }_{-0.017}^{+0.004}$ \\
\hline 15,40 & 0.016 & \pm 0.005 & $\begin{array}{l}+0.004 \\
-0.003\end{array}$ \\
\hline 40,1000 & 0.00025 & \pm 0.00010 & $\begin{array}{l}+0.00008 \\
{ }_{-0.0004} \\
\end{array}$ \\
\hline$x$ bin & $d \sigma / d x$ & $\begin{array}{c}\Delta_{\text {stat }} \\
(\mathrm{nb})\end{array}$ & $\Delta_{\text {syst }}$ \\
\hline $0.000021,0.0001$ & 4982 & \pm 1967 & $\begin{array}{l}+1354 \\
-1333\end{array}$ \\
\hline $0.0001, \quad 0.0005$ & 2765 & \pm 443 & $\begin{array}{l}+65 \\
-644\end{array}$ \\
\hline $0.0005, \quad 0.001$ & 934 & \pm 250 & ${ }_{-155}^{+118}$ \\
\hline $0.001,0.1$ & 6.1 & \pm 1.5 & $\begin{array}{l}+0.8 \\
{ }_{-0.6}^{+} \\
\end{array}$ \\
\hline$p_{T}\left(D_{s}^{+}\right)$bin $(\mathrm{GeV})$ & $d \sigma / d p_{T}\left(D_{s}^{+}\right)$ & $\begin{array}{c}\Delta_{\text {stat }} \\
(\mathrm{nb} / \mathrm{GeV})\end{array}$ & $\Delta_{\text {syst }}$ \\
\hline $2.0,2.5$ & 1.65 & \pm 0.52 & $\begin{array}{l}{ }_{-0.50}^{+0.36} \\
\quad\end{array}$ \\
\hline $2.5,3.0$ & 0.62 & \pm 0.22 & $\begin{array}{l}+0.14 \\
-0.11\end{array}$ \\
\hline $3.0,3.5$ & 0.59 & \pm 0.21 & $\begin{array}{l}+0.08 \\
-0.12\end{array}$ \\
\hline $3.5,4.5$ & 0.55 & \pm 0.11 & $\begin{array}{l}+0.05 \\
{ }_{-0.05}\end{array}$ \\
\hline $4.5,6.0$ & 0.20 & \pm 0.05 & $\begin{array}{l}+0.02 \\
-0.01\end{array}$ \\
\hline $6.0,20$. & 0.011 & \pm 0.004 & $\begin{array}{l}+0.002 \\
-0.001 \\
\end{array}$ \\
\hline$\eta\left(D_{s}^{+}\right)$bin & $d \sigma / d \eta\left(D_{s}^{+}\right)$ & $\begin{array}{l}\Delta_{\text {stat }} \\
(\mathrm{nb})\end{array}$ & $\Delta_{\text {syst }}$ \\
\hline$-1.6,-0.6$ & 0.94 & \pm 0.24 & $\begin{array}{l}+0.11 \\
-0.26\end{array}$ \\
\hline$-0.6, \quad 0.0$ & 0.57 & \pm 0.15 & $\begin{array}{l}+0.14 \\
-0.04\end{array}$ \\
\hline $0.0,0.6$ & 0.76 & \pm 0.18 & $\begin{array}{l}+0.06 \\
-0.09\end{array}$ \\
\hline $0.6,1.6$ & 0.85 & \pm 0.22 & $\begin{array}{l}+0.17 \\
-0.12\end{array}$ \\
\hline
\end{tabular}

Table 7: Measured $D_{s}^{+}$differential cross sections as a function of $Q^{2}, x, p_{T}\left(D_{s}^{+}\right)$ and $\eta\left(D_{s}^{+}\right)$for $1.5<Q^{2}<1000 \mathrm{GeV}^{2}, 0.02<y<0.7, p_{T}\left(D_{s}^{+}\right)>2 \mathrm{GeV}$ and $\left|\eta\left(D_{s}^{+}\right)\right|<1.6$. The estimated b-quark contribution of $4.3 \%$ has been subtracted. The statistical and systematic uncertainties are shown separately. The cross sections have a further $13 \%$ uncertainty from the $D_{s}^{+} \rightarrow \phi \pi^{+} \rightarrow K^{+} K^{-} \pi^{+}$branching ratio. 


\begin{tabular}{|c|c|c|c|c|c|c|c|c|c|c|}
\hline & $\begin{array}{c}\delta_{1}-\delta_{9} \\
(\%)\end{array}$ & $\begin{array}{c}\delta_{1}-\delta_{8} \\
(\%)\end{array}$ & $\begin{array}{c}\delta_{1} \\
(\%) \\
\end{array}$ & $\begin{array}{c}\delta_{2} \\
(\%) \\
\end{array}$ & $\begin{array}{c}\delta_{3} \\
(\%) \\
\end{array}$ & $\begin{array}{c}\delta_{4} \\
(\%) \\
\end{array}$ & $\begin{array}{c}\delta_{5} \\
(\%) \\
\end{array}$ & $\begin{array}{c}\delta_{6} \\
(\%) \\
\end{array}$ & $\begin{array}{c}\delta_{7} \\
(\%) \\
\end{array}$ & $\begin{array}{c}\delta_{8} \\
(\%) \\
\end{array}$ \\
\hline$\sigma^{\text {untag }}\left(D^{0}\right)$ & $\begin{array}{l}+5.8 \\
-4.7 \\
\end{array}$ & $\begin{array}{l}+5.4 \\
-4.1 \\
\end{array}$ & $\begin{array}{l}+2.5 \\
-0.0 \\
\end{array}$ & $\begin{array}{l}+1.7 \\
-3.3 \\
\end{array}$ & $\begin{array}{l}+1.3 \\
-0.9 \\
\end{array}$ & $\begin{array}{l}+1.5 \\
-0.6 \\
\end{array}$ & $\begin{array}{l}+3.5 \\
-0.4 \\
\end{array}$ & $\begin{array}{l}+2.2 \\
-2.1 \\
\end{array}$ & & \\
\hline$\sigma\left(D^{+}\right)$ & $\begin{array}{l}+6.6 \\
-5.0 \\
\end{array}$ & $\begin{array}{l}+3.2 \\
-4.6 \\
\end{array}$ & $\begin{array}{l}+0.6 \\
-0.0 \\
\end{array}$ & $\begin{array}{l}+1.5 \\
-3.1 \\
\end{array}$ & $\begin{array}{l}+1.0 \\
-1.9 \\
\end{array}$ & $\begin{array}{l}+1.4 \\
-0.6 \\
\end{array}$ & $\begin{array}{l}+0.0 \\
-1.5 \\
\end{array}$ & $\begin{array}{l}+2.3 \\
-2.2 \\
\end{array}$ & & \\
\hline$\sigma_{2}\left(D_{s}^{+}\right)$ & $\begin{array}{l}+9.1 \\
-7.4 \\
\end{array}$ & $\begin{array}{l}+8.8 \\
-7.2 \\
\end{array}$ & $\begin{array}{l}+0.0 \\
-2.0 \\
\end{array}$ & $\begin{array}{l}+0.0 \\
-4.0 \\
\end{array}$ & $\begin{array}{l}+1.2 \\
-0.0 \\
\end{array}$ & $\begin{array}{l}+0.5 \\
-0.4 \\
\end{array}$ & $\begin{array}{l}+8.0 \\
-5.1 \\
\end{array}$ & $\begin{array}{l}+2.2 \\
-2.1 \\
\end{array}$ & & \\
\hline$\sigma^{\operatorname{tag}}\left(D^{0}\right)$ & & $\begin{array}{l}+5.6 \\
-4.6 \\
\end{array}$ & $\begin{array}{l}+0.0 \\
-1.5 \\
\end{array}$ & $\begin{array}{l}+1.8 \\
-3.5 \\
\end{array}$ & $\begin{array}{l}+2.3 \\
-1.1 \\
\end{array}$ & $\begin{array}{l}+1.5 \\
-0.3 \\
\end{array}$ & $\begin{array}{l}+4.0 \\
-0.8 \\
\end{array}$ & $\begin{array}{l}+2.2 \\
-2.1 \\
\end{array}$ & & \\
\hline$\sigma^{\operatorname{add}}\left(D^{*+}\right)$ & & $\begin{array}{l}+9.9 \\
-8.9\end{array}$ & $\begin{array}{l}+0.0 \\
-2.9\end{array}$ & $\begin{array}{l}+1.9 \\
-3.8\end{array}$ & $\begin{array}{l}+4.2 \\
-0.2\end{array}$ & $\begin{array}{l}+2.3 \\
-0.5\end{array}$ & $\begin{array}{l}+8.2 \\
-7.2\end{array}$ & $\begin{array}{l}+2.2 \\
-2.1\end{array}$ & & \\
\hline$\sigma^{\operatorname{kin}}\left(D^{*+}\right)$ & & $\begin{array}{l}+5.7 \\
-4.7 \\
\end{array}$ & $\begin{array}{l}+0.0 \\
-1.8 \\
\end{array}$ & $\begin{array}{l}+1.8 \\
-3.6 \\
\end{array}$ & $\begin{array}{l}+2.5 \\
-0.8 \\
\end{array}$ & $\begin{array}{l}+1.6 \\
-0.3 \\
\end{array}$ & $\begin{array}{l}+4.0 \\
-1.1 \\
\end{array}$ & $\begin{array}{l}+2.2 \\
-2.1 \\
\end{array}$ & & \\
\hline$\sigma\left(D_{s}^{+}\right)$ & & $\begin{array}{l}+8.9 \\
-4.9 \\
\end{array}$ & $\begin{array}{l}+2.8 \\
-0.0 \\
\end{array}$ & $\begin{array}{l}+2.2 \\
-4.5 \\
\end{array}$ & $\begin{array}{l}+4.0 \\
-0.0 \\
\end{array}$ & $\begin{array}{l}+0.4 \\
-0.1\end{array}$ & $\begin{array}{l}+6.8 \\
-0.0 \\
\end{array}$ & $\begin{array}{l}+2.2 \\
-2.1 \\
\end{array}$ & & \\
\hline$R_{u / d}$ & & $\begin{array}{l}+4.3 \\
-1.4\end{array}$ & $\begin{array}{l}+2.7 \\
-0.0\end{array}$ & $\begin{array}{l}+0.0 \\
-0.1\end{array}$ & $\begin{array}{l}+1.4 \\
-1.4\end{array}$ & $\begin{array}{l}+0.1 \\
-0.1\end{array}$ & $\begin{array}{l}+3.0 \\
-0.3 \\
\end{array}$ & $\begin{array}{l}+0.1 \\
-0.1\end{array}$ & & \\
\hline$\gamma_{s}$ & & $\begin{array}{l}+7.9 \\
-3.0 \\
\end{array}$ & $\begin{array}{l}+1.8 \\
-0.0 \\
\end{array}$ & $\begin{array}{l}+0.5 \\
-1.2 \\
\end{array}$ & $\begin{array}{l}+4.1 \\
-0.4 \\
\end{array}$ & $\begin{array}{l}+0.6 \\
-1.3 \\
\end{array}$ & $\begin{array}{l}+6.4 \\
-2.4 \\
\end{array}$ & $\begin{array}{l}+0.0 \\
-0.0 \\
\end{array}$ & & \\
\hline$P_{\mathrm{v}}^{d}$ & & $\begin{array}{l}+2.8 \\
-1.4 \\
\end{array}$ & $\begin{array}{l}+0.0 \\
-1.3 \\
\end{array}$ & $\begin{array}{l}+0.1 \\
-0.3 \\
\end{array}$ & $\begin{array}{l}+1.4 \\
-0.0\end{array}$ & $\begin{array}{l}+0.2 \\
-0.0\end{array}$ & $\begin{array}{l}+2.4 \\
-0.0\end{array}$ & $\begin{array}{l}+0.0 \\
-0.1\end{array}$ & & \\
\hline$\sigma_{\mathrm{gs}}$ & & $\begin{array}{l}+10.3 \\
-4.3 \\
\end{array}$ & $\begin{array}{l}+1.2 \\
-0.0 \\
\end{array}$ & $\begin{array}{l}+1.7 \\
-3.4 \\
\end{array}$ & $\begin{array}{l}+1.2 \\
-0.5 \\
\end{array}$ & $\begin{array}{l}+1.4 \\
-0.5 \\
\end{array}$ & $\begin{array}{l}+2.4 \\
-0.5 \\
\end{array}$ & $\begin{array}{l}+2.2 \\
-2.1 \\
\end{array}$ & $\begin{array}{l}+9.3 \\
-0.9 \\
\end{array}$ & $\begin{array}{l}+0.4 \\
-0.4 \\
\end{array}$ \\
\hline$f\left(c \rightarrow D^{+}\right)$ & & $\begin{array}{l}+1.1 \\
-9.2 \\
\end{array}$ & $\begin{array}{l}+0.0 \\
-0.7 \\
\end{array}$ & $\begin{array}{l}+0.3 \\
-0.2 \\
\end{array}$ & $\begin{array}{l}+0.1 \\
-1.7 \\
\end{array}$ & $\begin{array}{l}+0.1 \\
-0.2 \\
\end{array}$ & $\begin{array}{l}+0.2 \\
-2.9 \\
\end{array}$ & $\begin{array}{l}+0.1 \\
-0.1 \\
\end{array}$ & $\begin{array}{l}+0.9 \\
-8.5 \\
\end{array}$ & $\begin{array}{l}+0.4 \\
-0.4 \\
\end{array}$ \\
\hline$f\left(c \rightarrow D^{0}\right)$ & & $\begin{array}{l}+1.1 \\
-8.6\end{array}$ & $\begin{array}{l}+0.0 \\
-0.0\end{array}$ & $\begin{array}{l}+0.0 \\
-0.0\end{array}$ & $\begin{array}{l}+0.5 \\
-0.4\end{array}$ & $\begin{array}{l}+0.2 \\
-0.1\end{array}$ & $\begin{array}{l}+1.8 \\
-0.0\end{array}$ & $\begin{array}{l}+0.0 \\
-0.0\end{array}$ & $\begin{array}{l}+0.9 \\
-8.5\end{array}$ & $\begin{array}{l}+0.4 \\
-0.4\end{array}$ \\
\hline$f^{\text {untag }}\left(c \rightarrow D^{0}\right)$ & & $\begin{array}{l}+2.0 \\
-8.6 \\
\end{array}$ & $\begin{array}{l}+1.0 \\
-0.0 \\
\end{array}$ & $\begin{array}{l}+0.1 \\
-0.0 \\
\end{array}$ & $\begin{array}{l}+0.5 \\
-0.9 \\
\end{array}$ & $\begin{array}{l}+0.2 \\
-0.1\end{array}$ & $\begin{array}{l}+1.4 \\
-0.2 \\
\end{array}$ & $\begin{array}{l}+0.0 \\
-0.0 \\
\end{array}$ & $\begin{array}{l}+0.9 \\
-8.5 \\
\end{array}$ & $\begin{array}{l}+0.4 \\
-0.4 \\
\end{array}$ \\
\hline$f\left(c \rightarrow D_{s}^{+}\right)$ & & $\begin{array}{l}+7.1 \\
-8.9 \\
\end{array}$ & $\begin{array}{l}+1.6 \\
-0.0 \\
\end{array}$ & $\begin{array}{l}+0.5 \\
-1.0 \\
\end{array}$ & $\begin{array}{l}+3.7 \\
-0.3 \\
\end{array}$ & $\begin{array}{l}+0.5 \\
-1.2 \\
\end{array}$ & $\begin{array}{l}+5.7 \\
-2.1 \\
\end{array}$ & $\begin{array}{l}+0.0 \\
-0.0 \\
\end{array}$ & $\begin{array}{l}+0.9 \\
-8.5 \\
\end{array}$ & $\begin{array}{l}+0.4 \\
-0.4 \\
\end{array}$ \\
\hline$f\left(c \rightarrow D^{*+}\right)$ & & $\begin{array}{l}+2.8 \\
-9.1 \\
\end{array}$ & $\begin{array}{l}+0.0 \\
-2.9 \\
\end{array}$ & $\begin{array}{l}+0.1 \\
-0.1 \\
\end{array}$ & $\begin{array}{l}+1.5 \\
-0.3 \\
\end{array}$ & $\begin{array}{l}+0.2 \\
-0.0 \\
\end{array}$ & $\begin{array}{l}+2.2 \\
-1.5 \\
\end{array}$ & $\begin{array}{l}+0.0 \\
-0.0 \\
\end{array}$ & $\begin{array}{l}+0.9 \\
-8.5 \\
\end{array}$ & $\begin{array}{l}+0.4 \\
-0.4 \\
\end{array}$ \\
\hline
\end{tabular}

Table 8: The systematic uncertainties resulting from $\delta_{1}-\delta_{9}$, from $\delta_{1}-\delta_{8}$, and from $\delta_{1}$ to $\delta_{8}$ independently (see text) for the charm hadron cross sections and charm fragmentation ratios and fractions. 
untagged $D^{0}$

\begin{tabular}{|c|c|cccc|}
\hline$Q^{2}$ bin $\left(\mathrm{GeV}^{2}\right)$ & $y$ bin & $\sigma$ & $\Delta_{\text {stat }}$ & $\Delta_{\text {syst }}$ & $(\mathrm{nb})$ \\
\hline \hline $1.5,9$ & $0.18,0.70$ & 1.50 & \pm 0.19 & ${ }_{-0.16}^{+0.14}$ & \\
& $0.02,0.18$ & 1.03 & \pm 0.19 & ${ }_{-0.12}^{+0.16}$ & \\
\hline 9,44 & $0.20,0.70$ & 1.02 & \pm 0.14 & ${ }_{-0.09}^{+0.12}$ & \\
& $0.02,0.20$ & 1.02 & \pm 0.14 & ${ }_{-0.05}^{+0.12}$ & \\
\hline 44,1000 & $0.02,0.70$ & 1.03 & \pm 0.19 & ${ }_{-0.05}^{+0.16}$ & \\
\hline
\end{tabular}

\begin{tabular}{|c|c|cccc|}
\multicolumn{9}{|c|}{$D^{+}$} & & & \\
\hline$Q^{2}$ bin $\left(\mathrm{GeV}^{2}\right)$ & $y$ bin & $\sigma$ & $\Delta_{\text {stat }}$ & $\Delta_{\text {syst }}$ & $(\mathrm{nb})$ \\
\hline \hline $1.5,9$ & $0.18,0.70$ & 0.63 & \pm 0.14 & ${ }_{-0.10}^{+0.05}$ & \\
& $0.02,0.18$ & 0.65 & \pm 0.13 & ${ }_{-0.08}^{+0.09}$ & \\
\hline 9,44 & $0.20,0.70$ & 0.52 & \pm 0.11 & ${ }_{-0.10}^{+0.03}$ & \\
& $0.02,0.20$ & 0.44 & \pm 0.11 & ${ }_{-0.03}^{+0.06}$ & \\
\hline 44,1000 & $0.02,0.70$ & 0.61 & \pm 0.25 & ${ }_{-0.09}^{+0.08}$ & \\
\hline
\end{tabular}

\begin{tabular}{|c|c|cccc|}
\multicolumn{9}{c|}{$D_{s}^{+}$} & & \\
\hline$Q^{2} \operatorname{bin}\left(\mathrm{GeV}^{2}\right)$ & $y$ bin & $\sigma$ & $\Delta_{\text {stat }}$ & $\Delta_{\text {syst }}$ & $(\mathrm{nb})$ \\
\hline \hline $1.5,9$ & $0.18,0.70$ & 0.97 & \pm 0.23 & ${ }_{-0.21}^{+0.16}$ & \\
& $0.02,0.18$ & 0.56 & \pm 0.15 & ${ }_{-0.08}^{+0.11}$ & \\
\hline 9,44 & $0.20,0.70$ & 0.61 & \pm 0.15 & ${ }_{-0.15}^{+0.14}$ & \\
& $0.02,0.20$ & 0.29 & \pm 0.07 & ${ }_{-0.03}^{+0.12}$ & \\
\hline 44,1000 & $0.02,0.70$ & 0.20 & \pm 0.11 & ${ }_{-0.03}^{+0.08}$ & \\
\hline
\end{tabular}

Table 9: $\quad$ Measured cross sections for $D^{0}$ not coming from a $D^{*+}, D^{+}$and $D_{s}^{+}$ in each of the $Q^{2}$ and $y$ bins for $p_{T}\left(D^{0}, D^{+}\right)>3 \mathrm{GeV}, p_{T}\left(D_{s}^{+}\right)>2 \mathrm{GeV}$ and $|\eta(D)|<1.6$. The estimated b-quark contribution of $3.1 \%$ for $D^{0}$ and $D^{+}$and $4.3 \%$ for $D_{s}^{+}$has been subtracted. The statistical and systematic uncertainties are shown separately. The $D^{0}, D^{+}$and $D_{s}^{+}$cross sections have further $1.8 \%, 3.6 \%$ and $13 \%$ uncertainties from the $D^{0} \rightarrow K^{-} \pi^{+}, D^{+} \rightarrow K^{-} \pi^{+} \pi^{+}$and $D_{s}^{+} \rightarrow \phi \pi^{+} \rightarrow K^{+} K^{-} \pi^{+}$ branching ratios, respectively. 
untagged $D^{0}$

\begin{tabular}{|c|c|ccc|cc|}
\hline$Q^{2}\left(\mathrm{GeV}^{2}\right)$ & $x$ & $F_{2}^{c \bar{c}}$ & $\Delta_{\text {stat }}$ & $\Delta_{\text {syst }}$ & $\Delta_{\text {extrap }}$ & factor \\
\hline \hline 4.2 & 0.00013 & 0.141 & \pm 0.017 & ${ }_{-0.015}^{+0.013}$ & ${ }_{-0.013}^{+0.048}$ & 8.9 \\
& 0.00061 & 0.090 & \pm 0.017 & ${ }_{-0.011}^{+0.014}$ & ${ }_{-0.006}^{+0.036}$ & 17 \\
\hline 20.4 & 0.00062 & 0.320 & \pm 0.044 & ${ }_{-0.029}^{+0.037}$ & ${ }_{-0.020}^{+0.061}$ & 4.9 \\
& 0.00281 & 0.156 & \pm 0.021 & ${ }_{-0.008}^{+0.019}$ & ${ }_{-0.004}^{+0.041}$ & 5.8 \\
\hline 111.8 & 0.00676 & 0.217 & \pm 0.039 & ${ }_{-0.011}^{+0.033}$ & ${ }_{-0.002}^{+0.014}$ & 2.5 \\
\hline
\end{tabular}

$D^{+}$

\begin{tabular}{|c|c|ccc|cc|}
\hline$Q^{2}\left(\mathrm{GeV}^{2}\right)$ & $x$ & $F_{2}^{c \bar{c}}$ & $\Delta_{\text {stat }}$ & $\Delta_{\text {syst }}$ & $\Delta_{\text {extrap }}$ & factor \\
\hline \hline 4.2 & 0.00013 & 0.123 & \pm 0.025 & ${ }_{-0.020}^{+0.010}$ & ${ }_{-0.011}^{+0.037}$ & 8.9 \\
& 0.00061 & 0.109 & \pm 0.020 & ${ }_{-0.014}^{+0.015}$ & ${ }_{-0.007}^{+0.039}$ & 17 \\
\hline 20.4 & 0.00062 & 0.331 & \pm 0.067 & ${ }_{-0.067}^{+0.016}$ & ${ }_{-0.021}^{+0.066}$ & 4.9 \\
& 0.00281 & 0.130 & \pm 0.039 & ${ }_{-0.009}^{+0.017}$ & ${ }_{-0.003}^{+0.030}$ & 5.8 \\
\hline 111.8 & 0.00676 & 0.293 & \pm 0.124 & ${ }_{-0.041}^{+0.037}$ & ${ }_{-0.003}^{+0.021}$ & 2.5 \\
\hline
\end{tabular}

\begin{tabular}{|c|c|ccc|cc|}
\multicolumn{7}{c|}{$D_{s}^{+}$} \\
\hline$Q^{2}\left(\mathrm{GeV}^{2}\right)$ & $x$ & $F_{2}^{c \bar{c}}$ & $\Delta_{\text {stat }}$ & $\Delta_{\text {syst }}$ & $\Delta_{\text {extrap }}$ & factor \\
\hline \hline 4.2 & 0.00013 & 0.221 & \pm 0.044 & ${ }_{-0.048}^{+0.037}$ & ${ }_{-0.016}^{+0.036}$ & 4.3 \\
& 0.00061 & 0.075 & \pm 0.017 & ${ }_{-0.011}^{+0.016}$ & ${ }_{-0.004}^{+0.019}$ & 5.6 \\
\hline 20.4 & 0.00062 & 0.470 & \pm 0.100 & ${ }_{-0.112}^{+0.109}$ & ${ }_{-0.017}^{+0.037}$ & 2.8 \\
& 0.00281 & 0.100 & \pm 0.022 & ${ }_{-0.009}^{+0.043}$ & ${ }_{-0.001}^{+0.013}$ & 2.9 \\
\hline 111.8 & 0.00676 & 0.179 & \pm 0.058 & ${ }_{-0.025}^{+0.075}$ & ${ }_{-0.001}^{+0.013}$ & 1.9 \\
\hline
\end{tabular}

Combined

\begin{tabular}{|c|c|ccc|c|}
\hline$Q^{2}\left(\mathrm{GeV}^{2}\right)$ & $x$ & $F_{2}^{c \bar{c}}$ & $\Delta_{\text {stat }}$ & $\Delta_{\text {syst }}$ & $\Delta_{\text {extrap }}$ \\
\hline \hline 4.2 & 0.00013 & 0.144 & \pm 0.014 & ${ }_{-0.015}^{+0.022}$ & ${ }_{-0.013}^{+0.045}$ \\
& 0.00061 & 0.090 & \pm 0.010 & ${ }_{-0.004}^{+0.010}$ & ${ }_{-0.005}^{+0.029}$ \\
\hline 20.4 & 0.00062 & 0.341 & \pm 0.035 & ${ }_{-0.042}^{+0.046}$ & ${ }_{-0.021}^{+0.063}$ \\
& 0.00281 & 0.132 & \pm 0.014 & ${ }_{-0.005}^{+0.024}$ & ${ }_{-0.001}^{+0.024}$ \\
\hline 111.8 & 0.00676 & 0.211 & \pm 0.032 & ${ }_{-0.013}^{+0.044}$ & ${ }_{-0.002}^{+0.013}$ \\
\hline
\end{tabular}

Table 10: The extracted values of $F_{2}^{c \bar{c}}$ from the production cross sections of $D^{0}$ not coming from $D^{*+}, D^{+}$and $D_{s}^{+}$and the combination of them at each $Q^{2}$ and $x$ value. The statistical, systematic and extrapolation uncertainties are shown separately. The values of the extrapolation factor used to correct the full $p_{T}(D)$ and $\eta(D)$ phase space are also shown. All the extracted $F_{2}^{c \bar{c}}$ values have a further $+2.7 \%-4.1 \%$ uncertainty from the $D^{0} \rightarrow K^{-} \pi^{+}, D^{+} \rightarrow K^{-} \pi^{+} \pi^{+}$and $D_{s}^{+} \rightarrow \phi \pi^{+} \rightarrow K^{+} K^{-} \pi^{+}$ branching ratios and the $f\left(c \rightarrow \Lambda_{c}^{+}\right)$value. 

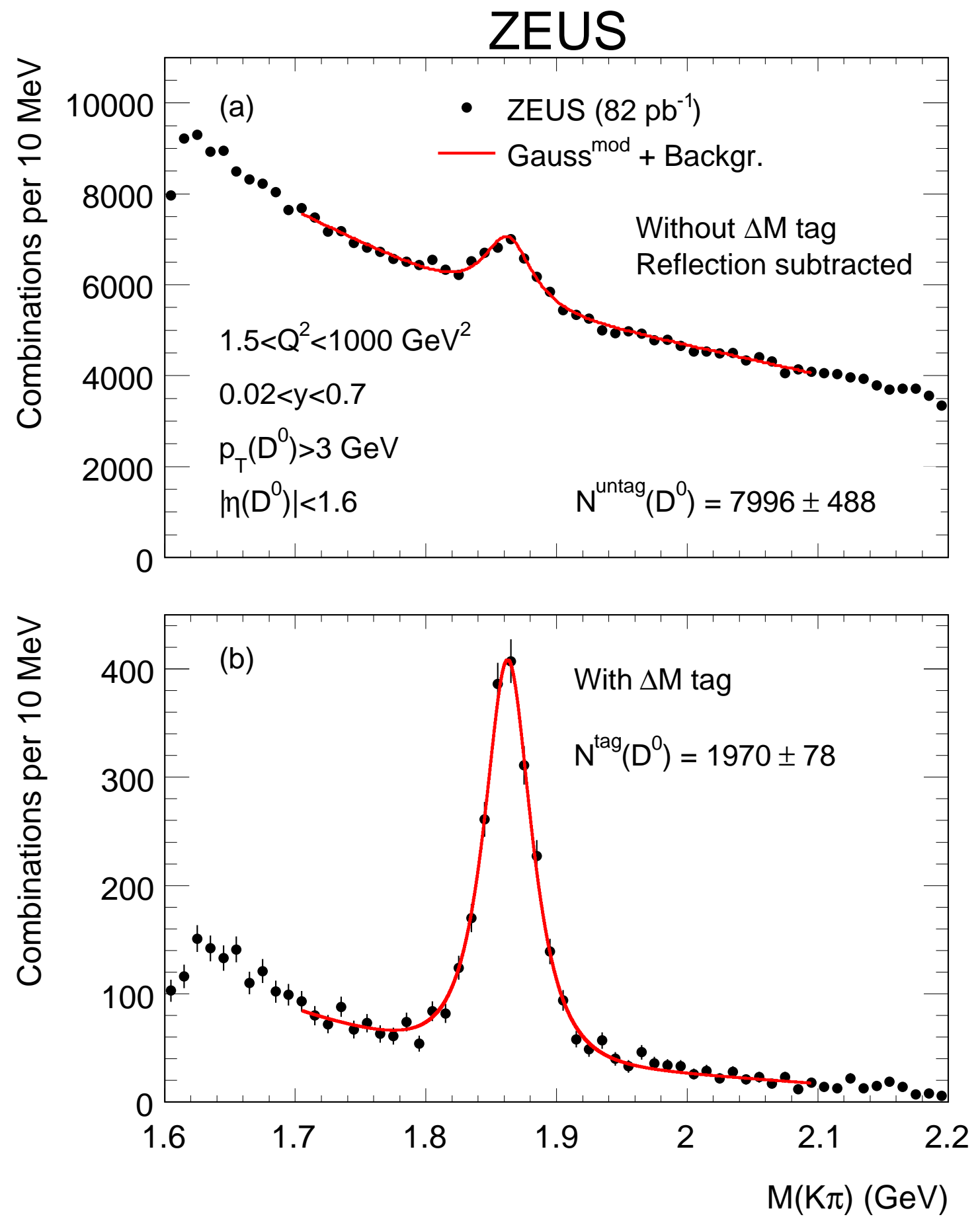

Figure 1: The $M\left(K^{-} \pi^{+}\right)$distributions (dots) for (a) the $D^{0}$ candidates without $\Delta M$ tag, obtained after reflection subtraction, and for $(b)$ the $D^{0}$ candidates with $\Delta M$ tag. The lowest and highest mass bins are affected by the trigger selection. The solid curves represent a fit to the sum of a modified Gaussian function and a background function. 


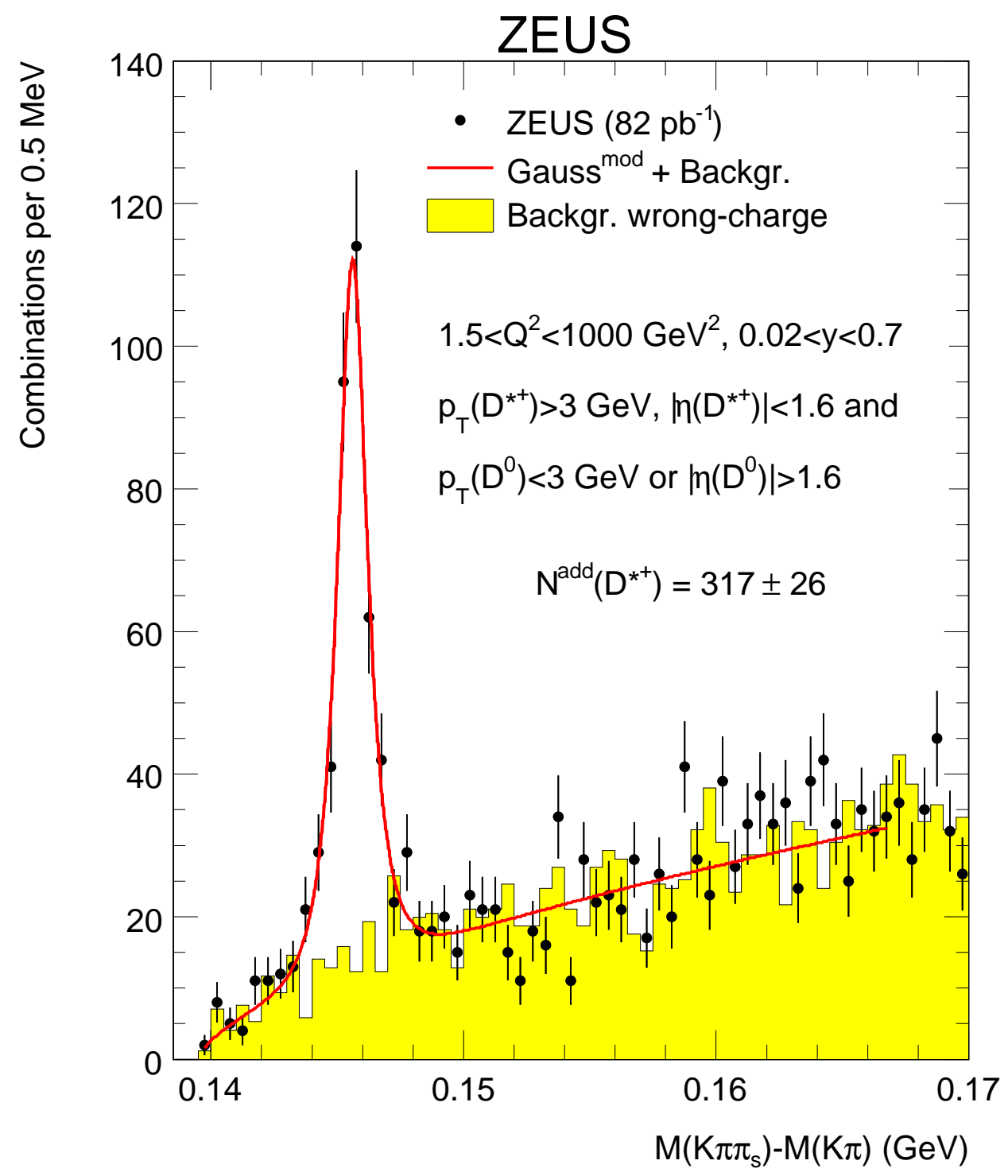

Figure 2: The distribution of the mass difference, $\Delta M=M\left(K^{-} \pi^{+} \pi_{s}^{+}\right)-$ $M\left(K^{-} \pi^{+}\right)$, for the "additional" $D^{*+}$ candidates (dots). The histogram shows the $\Delta M$ distribution for wrong-charge combinations. For illustration, the solid curve represents a fit to the sum of a modified Gaussian function and a background function. 


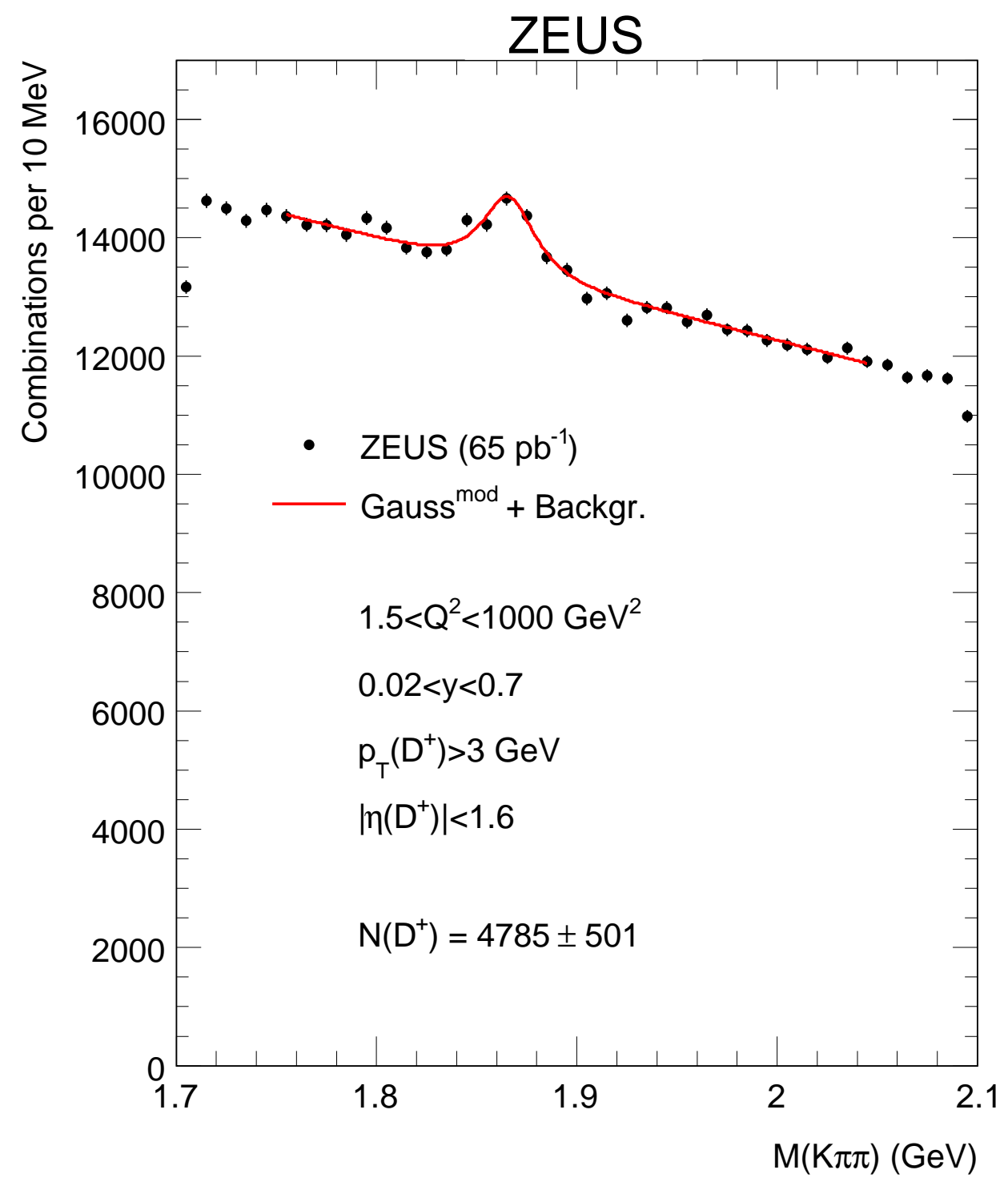

Figure 3: The $M\left(K^{-} \pi^{+} \pi^{+}\right)$distribution for the $D^{+}$candidates (dots). The lowest and highest mass bins are affected by the trigger selection. The solid curve represents a fit to the sum of a modified Gaussian function and a linear background function. 

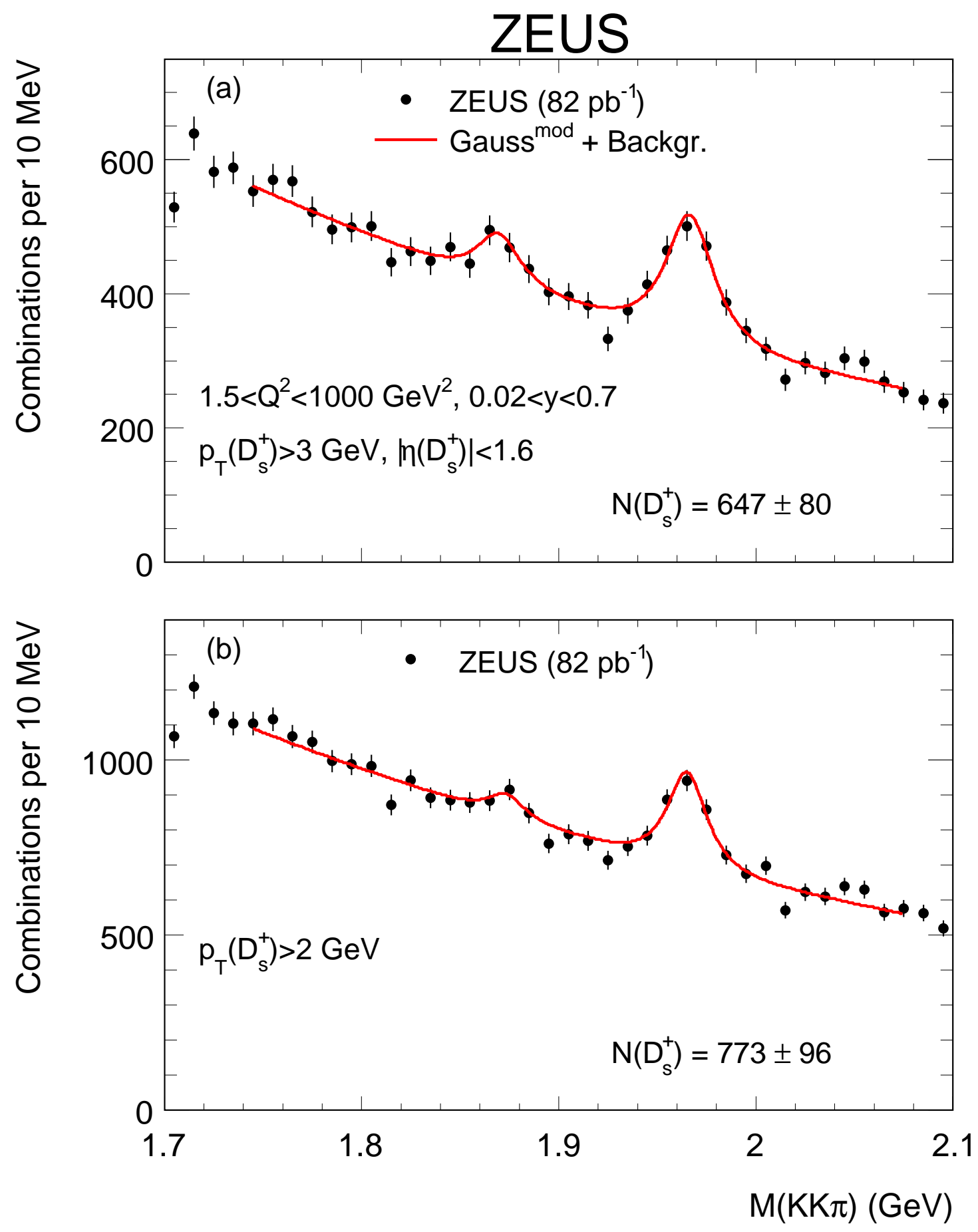

Figure 4: The $M\left(K^{+} K^{-} \pi^{+}\right)$distribution for (a) $D_{s}^{+}$candidates with $p_{T}\left(D_{s}^{+}\right)>3$ $G e V$ and (b) $D_{s}^{+}$candidates with $p_{T}\left(D_{s}^{+}\right)>2 \mathrm{GeV}$. The lowest mass bins are affected by the trigger selection. The solid curves represent fits to the sum of two modified Gaussian functions and an exponential background function. The first peak in both distributions is from $D^{+}$decaying through the same channel. 

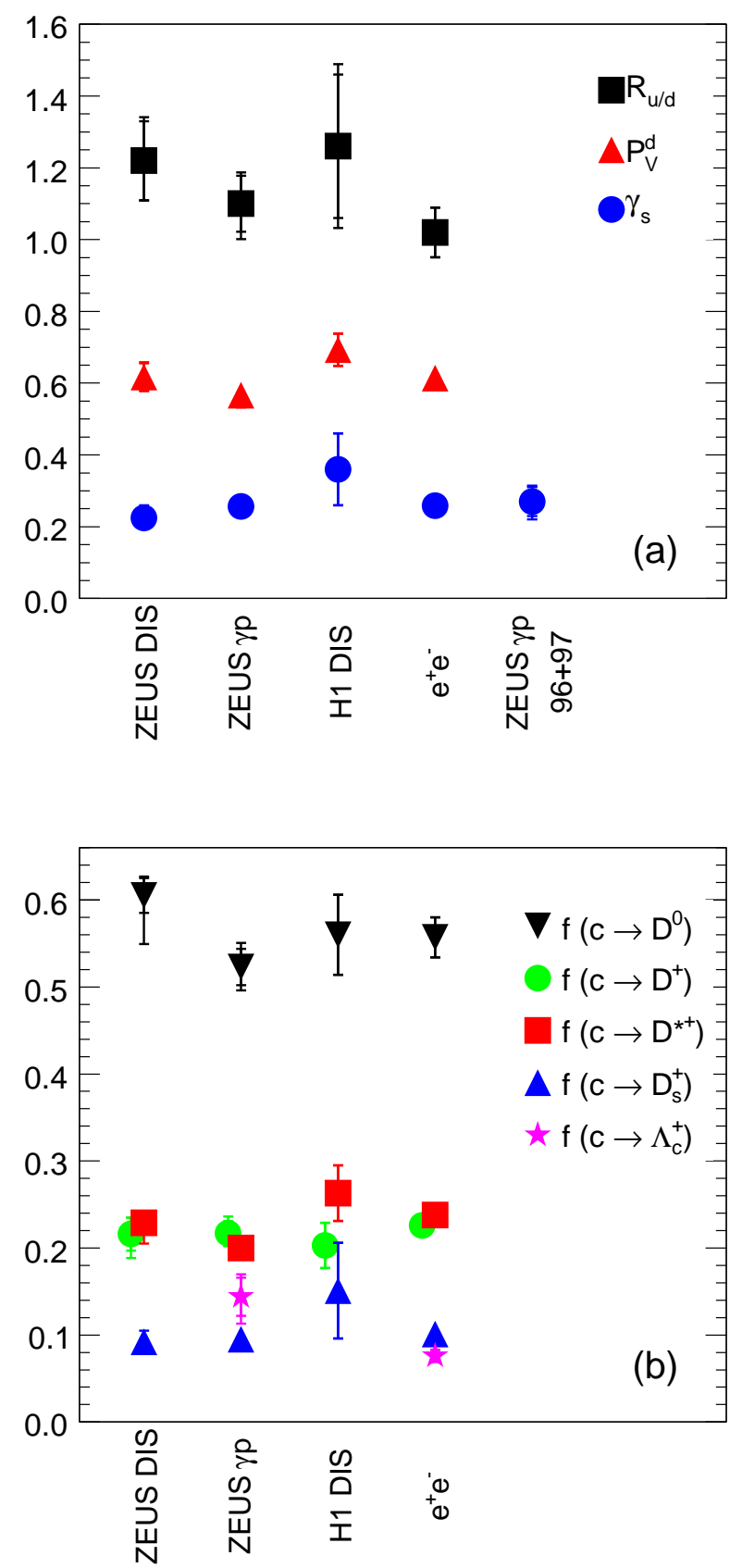

Figure 5: (a) The ratio of neutral to charged D-meson production rates, $R_{u / d}$, the strangeness-suppression factor in charm fragmentation, $\gamma_{s}$, and the fraction of charged $D$ mesons produced in a vector state, $P_{\mathrm{v}}^{d} \cdot$ (b) The fractions of c quarks hadronising as $D^{+}, D^{0}$ and $D_{s}^{+}$charm ground-state mesons, as $D^{*+}$ mesons and as $\Lambda_{c}^{+}$baryons. The inner error bars show the statistical uncertainties and the outer bars show the statistical and systematic uncertainties added in quadrature. The measurements have further uncertainties coming from the different branching ratios involved; their magnitudes are shown in Tables 2,3 and 4 for $R_{u / d}, \gamma_{s}$ and $P_{\mathrm{v}}^{d}$, respectively, and in Table 5 for the fractions. 


\section{ZEUS}
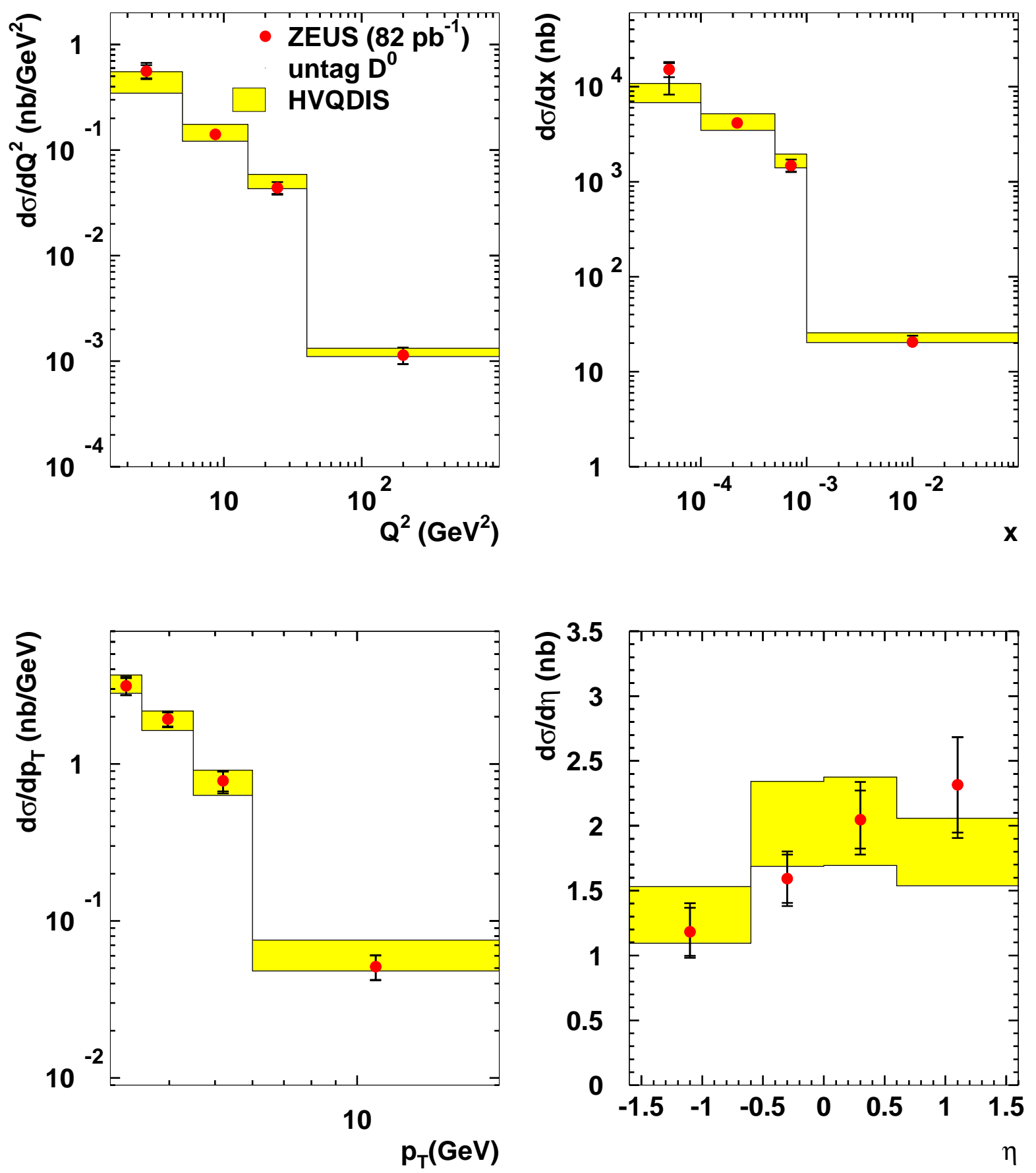

Figure 6: Differential cross sections for $D^{0}$ not coming from $D^{*+}$ as a function of $Q^{2}, x, p_{T}\left(D^{0}\right)$ and $\eta\left(D^{0}\right)$ compared to the NLO QCD calculation of HVQDIS. The inner error bars show the statistical uncertainties and the outer bars show the statistical and systematic uncertainties added in quadrature. The lower and upper NLO QCD predictions show the estimated theoretical uncertainty of the HVQDIS calculations. The data points have a further $1.8 \%$ uncertainty from the $D^{0} \rightarrow$ $K^{-} \pi^{+}$branching ratio. 


\section{ZEUS}
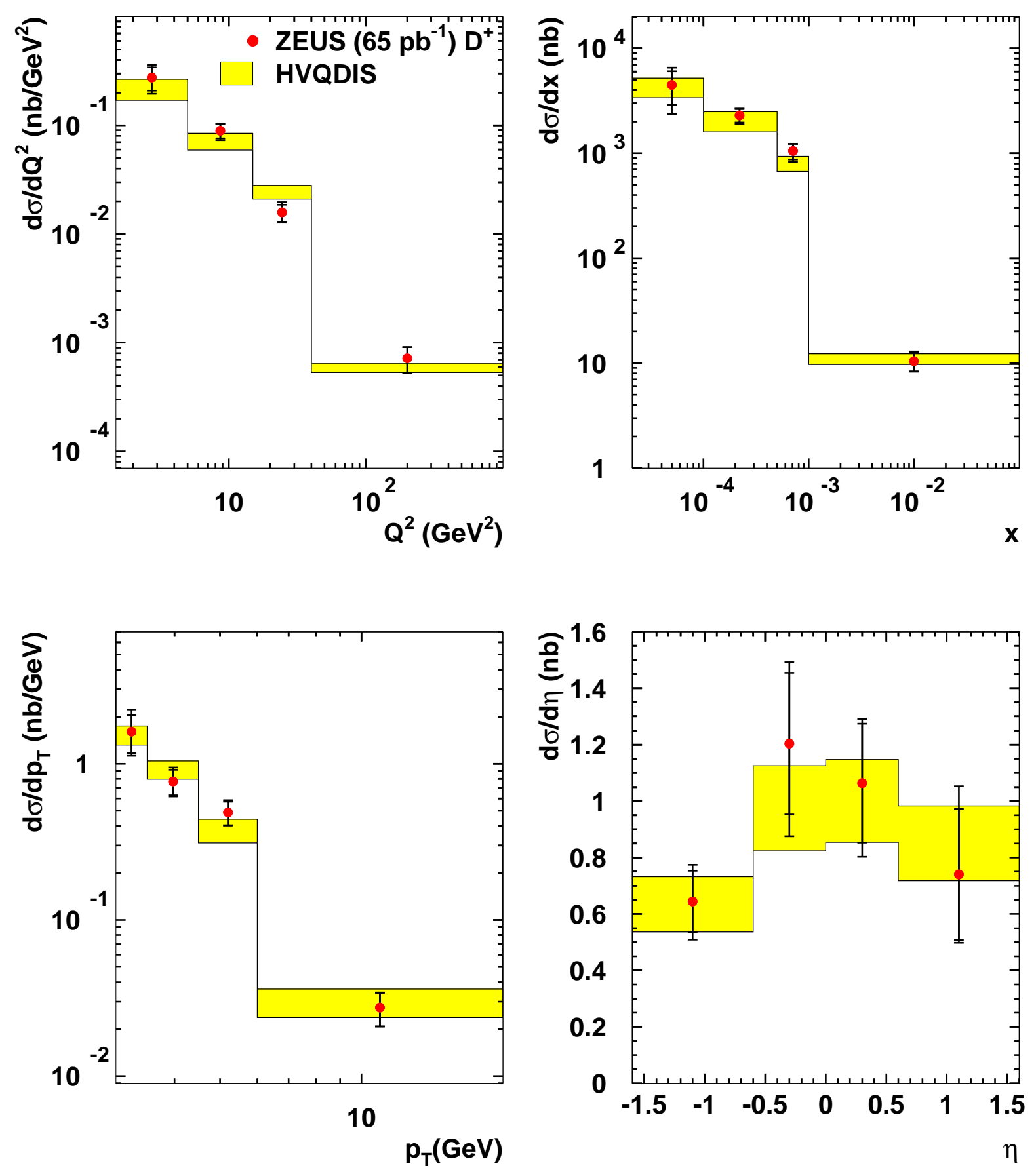

Figure 7: Differential $D^{+}$cross sections as a function of $Q^{2}, x, p_{T}\left(D^{+}\right)$and $\eta\left(D^{+}\right)$ compared to the NLO QCD calculation of HVQDIS. The inner error bars show the statistical uncertainties and the outer bars show the statistical and systematic uncertainties added in quadrature. The lower and upper NLO QCD predictions show the estimated theoretical uncertainty of the HVQDIS calculations. The data points have a further $3.6 \%$ uncertainty from the $D^{+} \rightarrow K^{-} \pi^{+} \pi^{+}$branching ratio. 


\section{ZEUS}
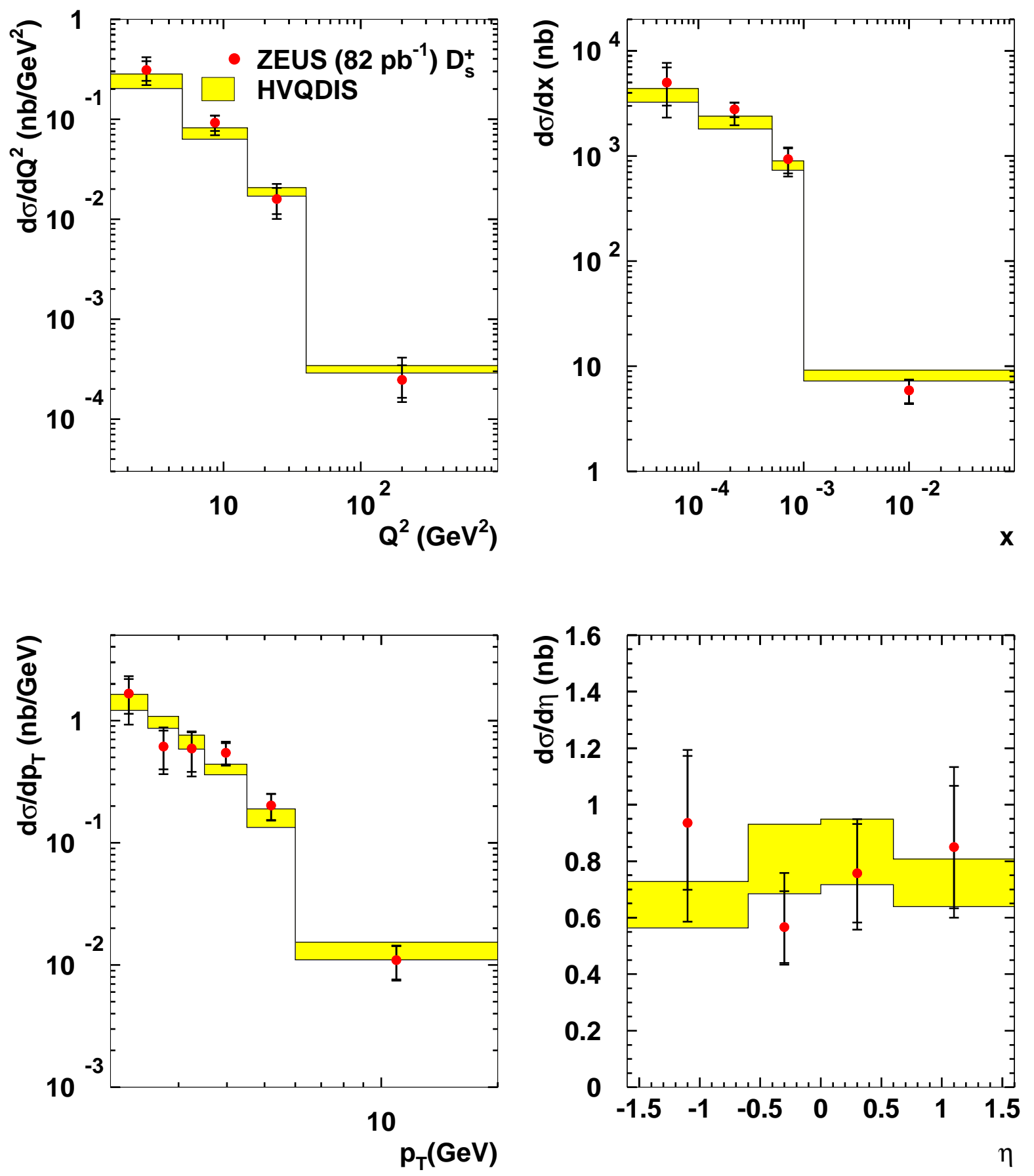

Figure 8: Differential $D_{s}^{+}$cross sections as a function of $Q^{2}, x, p_{T}\left(D_{s}^{+}\right)$and $\eta\left(D_{s}^{+}\right)$ compared to the NLO QCD calculation of HVQDIS. The inner error bars show the statistical uncertainties and the outer bars show the statistical and systematic uncertainties added in quadrature. The lower and upper NLO QCD predictions show the estimated theoretical uncertainty of the HVQDIS calculations. The data points have a further $13 \%$ uncertainty from the $D_{s}^{+} \rightarrow \phi \pi^{+} \rightarrow K^{+} K^{-} \pi^{+}$branching ratio. 


\section{ZEUS}
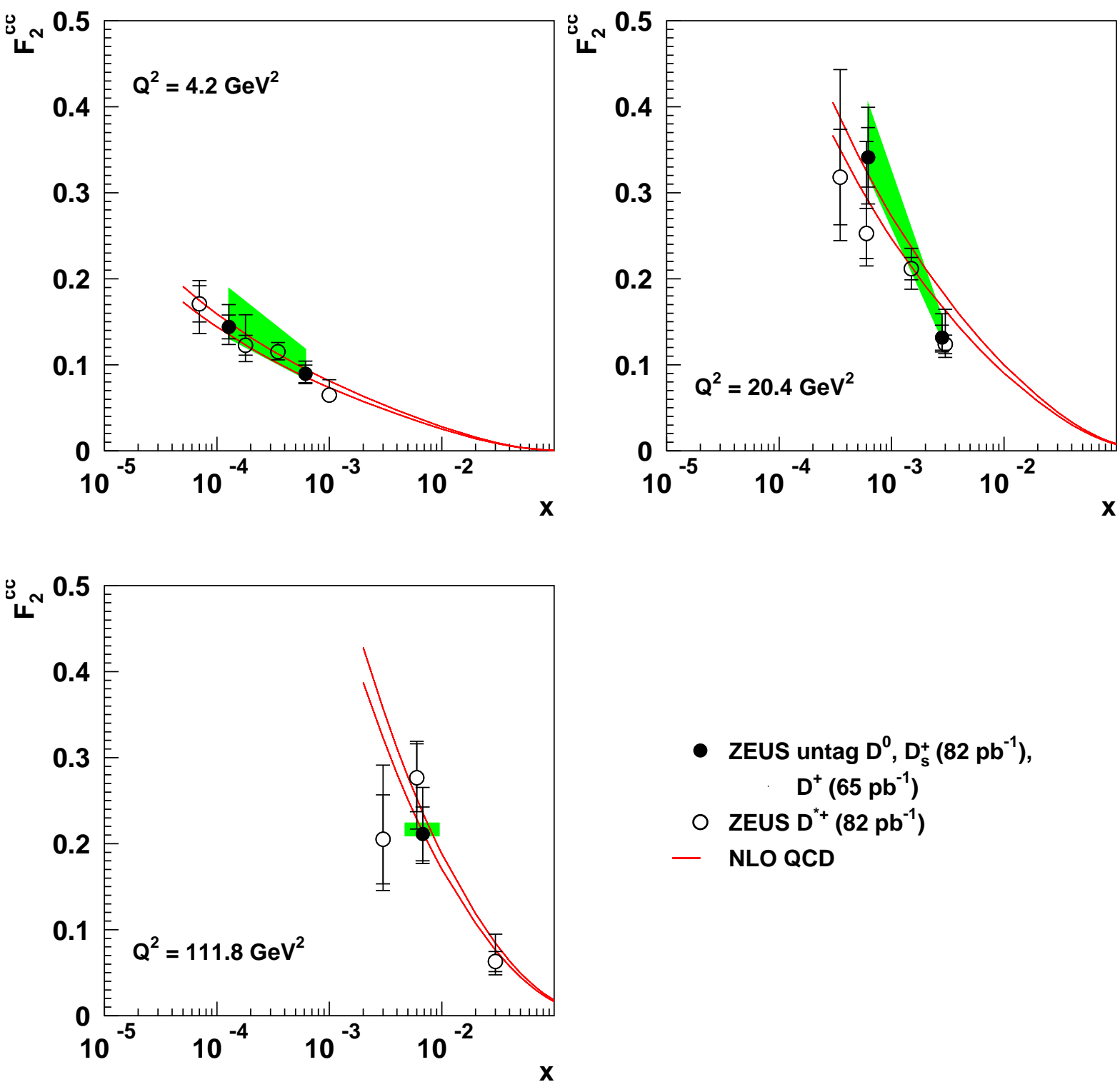

Figure 9: The measured $F_{2}^{c \bar{c}}$ as a function of $x$ for three $Q^{2}$ bins. The current data are compared with the previous ZEUS measurement [3]. The data are shown with statistical uncertainties (inner bars) and statistical and systematic uncertainties added in quadrature (outer bars). All measured $F_{2}^{c \bar{c}}$ values have a further +2.7\% -4.1\% uncertainty coming from the current experimental uncertainty from the $D^{0} \rightarrow K^{-} \pi^{+}, D^{+} \rightarrow K^{-} \pi^{+} \pi^{+}$and $D_{s}^{+} \rightarrow \phi \pi^{+} \rightarrow K^{+} K^{-} \pi^{+}$branching ratios and the $f\left(c \rightarrow \Lambda_{c}^{+}\right)$value. The shaded band corresponds to the estimated theoretical uncertainty in the extrapolation. The lower and upper curves show the ZEUS NLO QCD fit [27] uncertainty propagated from the experimental uncertainties of the fitted data. 